\title{
Carcinofauna do Complexo baía-estuário de Santos-São Vicente e canal de Bertioga, com destaque para Decapoda da Baía de Santos, São Paulo, Brasil
}

\author{
Carcinofauna of the Santos-São Vicente bay-estuary Complex and Bertioga channel, especially \\ Decapoda of Santos Bay, São Paulo, Brazil \\ Carcinofauna del Complejo de la bahía-estuario Santos-São Vicente y el canal Bertioga, destacando \\ Decapoda de la Bahía de Santos, São Paulo, Brasil
}

Jorge Luis dos Santos

ORCID: https://orcid.org/0000-0002-5858-0197

Universidade Santa Cecília, Brasil Universidade Federal de São Carlos, Brasil

E-mail: jorgepescabr@yahoo.com.br

Amanda Aparecida Carminatto ORCID: https://orcid.org/0000-0002-3765-7992 Universidade Federal de São Carlos, Brasil Núcleo de Tecnologia Marinha e Ambiental, Brasil

E-mail: amandacarminatto@gmail.com

Álvaro Luiz Diogo Reigada

ORCID: https://orcid.org/0000-0002-5842-5453

Universidade Santa Cecília, Brasil

E-mail: areigada@unisanta.br

Miguel Petrere Júnior

ORCID: https://orcid.org/0000-0003-2000-6699

Universidade Federal do Pará, Brasil

Universidade Federal de São Carlos, Brasil

Universidade Santa Cecília, Brasil

E-mail: mpetrerejr@gmail.com

\begin{abstract}
Resumo
Apesar da relevância geoeconômica da Baía de Santos, estudos sobre a composição de sua carcinofauna ainda são incipientes. Assim, este estudo fornece informações sobre a fauna de crustáceos da Baía de Santos, também copilando dados para Complexo baía-estuário de Santos-São Vicente e canal de Bertioga (CBESSB), localizado no litoral centro do estado de São Paulo. Foram realizadas quatro campanhas amostrais entre abril e dezembro de 2017 em seis pontos distintos, utilizando uma embarcação do tipo bote camaroeiro com esforço de captura padronizado. Também foi realizado um levantamento das espécies registradas no CBESSB. No total, foram registradas 102 espécies, pertencentes a 67 gêneros, 31 famílias, sete infraordens e três ordens. O monitoramento registrou um total de 13.404 exemplares (91.890,07 g), pertencentes a 18 espécies, 15 gêneros, 11 famílias, quatro infraordens e uma ordem. As espécies com a maior abundância numérica e em peso foram Xiphopenaeus kroyeri, Callinectes danae e Callinectes ornatus. No inverno, a faixa de média profundidade e o lado oeste da Baía de Santos apresentaram as maiores abundâncias. Apesar da antropização, o CBESSB apresenta elevada riqueza de crustáceos e sugere-se que seja monitorado constantemente com o objetivo de apoiar medidas de conservação e manejo, devido sua elevada importância ecológica, econômica e social para toda a região do litoral central do estado de São Paulo.
\end{abstract}

Palavras-chave: Inventário; Crustáceos; Abundância; Conservação.

\begin{abstract}
Despite the geo-economic relevance of the Santos Bay, studies on the composition of its carcinofauna are still incipient. Thus, this study provides information on the crustacean fauna of the Santos Bay, also compiling data for the SantosSão Vicente Bay-Estuary Complex and the Bertioga Canal (CBESSB), located on the central coast of the state of São Paulo. Four sampling campaigns were carried out between April and December 2017 at six different points, using a shrimp boat type with standardized capture effort. A survey of species registered with CBESSB was also carried out. In total, 102 species were registered, belonging to 67 genera, 31 families, seven infraordens and three orders. The monitoring recorded a total of 13,404 specimens $(91,890.07 \mathrm{~g})$, belonging to 18 species, 15 genera, 11 families, four infraordens and one order. The species with the highest numerical abundance and weight were Xiphopenaeus kroyeri, Callinectes danae and Callinectes ornatus. In winter, the medium depth strip and the west side of the Santos Bay presented the greatest abundance. Despite the anthropization, CBESSB has a high richness of crustaceans and it is
\end{abstract}


suggested that it be constantly monitored in order to support conservation and management measures, due to its high ecological, economic and social importance for the entire region of the central coast of the state of São Paulo.

Keywords: Inventory; Crustaceans; Abundance; Conservation.

\section{Resumen}

A pesar de la relevancia geoeconómica de la Bahía de Santos, los estudios sobre la composición de su carcinofauna son aún incipientes. Así, este estudio proporciona información sobre la fauna de crustáceos de la Bahía de Santos, compilando también datos para el Complejo Bahía-Estuario Santos-São Vicente y el Canal Bertioga (CBESSB), ubicados en la costa central del estado de São Paulo. Se realizaron cuatro campañas de muestreo entre abril y diciembre de 2017 en seis puntos diferentes, utilizando un tipo de barco camaronero con esfuerzo de captura estandarizado. También se llevó a cabo un estudio de especies registradas en CBESSB. En total se registraron 102 especies, pertenecientes a 67 géneros, 31 familias, siete infraorden y tres órdenes. El seguimiento registró un total de 13.404 ejemplares $(91.890,07 \mathrm{~g})$, pertenecientes a 18 especies, 15 géneros, 11 familias, cuatro infraordens y una orden. Las especies con mayor abundancia numérica y peso fueron Xiphopenaeus kroyeri, Callinectes danae y Callinectes ornatus. En invierno, la franja de profundidad media y el lado oeste de la Bahía de Santos presentaron la mayor abundancia. A pesar de la antropización, CBESSB tiene una alta riqueza de crustáceos y se sugiere que sea monitoreada constantemente para apoyar las medidas de conservación y manejo, debido a su alta importancia ecológica, económica y social para toda la región de la costa central del estado de São Paulo.

Palabras clave: Inventario; Crustáceos; Abundancia; Conservación.

\section{Introdução}

A Baixada Santista é um macro ecossistema que engloba vários ambientes costeiros, tais como estuários, manguezais, praias e ilhas, além de áreas de conservação, um expressivo complexo industrial e o maior porto da América Latina, resultando na área mais densamente povoada da zona costeira do estado de São Paulo. Assim, levantamentos contínuos das comunidades bióticas passíveis de alterações decorrentes das inúmeras atividades antrópicas são necessários (Abessa et al., 2008, Sousa, Zaroni, Gasparro, \& Pereira, 2014, Kim et al., 2016, Nunes, Greco, \& Marengo, 2019).

A região da Baía de Santos se insere nesse contexto pela elevada incidência antrópica, seja pelo transporte de contaminantes do estuário ou pela deposição do material de dragagem. O porto, a pesca e o turismo são atividades altamente impactantes que contaminam os sedimentos na região do alto estuário, Baía de Santos e adjacências, representando uma ameaça ao funcionamento harmônico dos ecossistemas aquáticos (Lamparelli et al., 2001, Abessa, 2002, Cesar et al., 2006, Buruaem, Hortellani, Sarkis, Costa-Lotufo, \& Abessa, 2012, Nunes et al., 2019).

Os organismos bentônicos, como os crustáceos, ficam expostos a sedimentos contaminados e assim, podem ter algumas de suas funções biológicas alteradas ou comprometidas. Consequentemente, estes contaminantes uma vez incorporados pelos organismos bentônicos podem atingir toda a cadeia trófica do ecossistema, afetando não somente a biota em contato direto com os sedimentos (Choueri, 2008, Cortez et al., 2018, Carvalho-Neta et al., 2019). É importante destacar que devido à baixa motilidade dos crustáceos, estes ficam sujeitos a maior exposição dos compostos presentes no fundo (sedimentos) e coluna d’água. Todavia, essas interações estão bem descritas (Choueri, 2008, Quintana, 2008), sendo necessários estudos que relacionem a estrutura da comunidade de crustáceos com ambientes impactados, como a Baía de Santos.

$\mathrm{Na}$ Baía de Santos ocorre um intenso esforço pesqueiro exercido pela frota de pequena escala (Gefe, Amorim, Amorim, \& Amorim, 2004). Segundo Reigada, Sant'anna, Zangrande e Costa (2006) a região apresenta ocorrência sazonal de espécies de interesse comercial como Callinectes sp. (siri-azul) e Penaeus schmitti Burkenroad, 1936 (camarão-branco), que juntamente com Xiphopenaeus kroyeri (Heller, 1862) (camarão sete-barbas) se destacam comercialmente na Baixada Santista (Santos, SeverinoRodrigues, \& Vaz-dos-Santos, 2008). Além da relevância econômica, segundo a atualização em vigor sobre as áreas prioritárias para a conservação da zona costeira e marinha brasileira (MMA, 2018), a região é caracterizada como de extrema importância biológica e com prioridade de ações, entre elas o inventário de sua biodiversidade.

Conforme no Decreto n ${ }^{\circ} 53.526$, de 8 de outubro de 2008, diferentes setores que formam a Área de Proteção Ambiental Marinha do Litoral Centro (APA Marinha do Litoral Centro), evidenciam a necessidade de se investir em levantamentos que 
visem aumentar o conhecimento dos fatores ecológicos e ambientais da região da Baía de Santos e adjacências. Principalmente no atual contexto de políticas públicas relacionadas à ampliação das atividades portuárias em Santos.

Estudos de levantamento da carcinofauna são importantes instrumentos para o desenvolvimento de políticas de conservação, auxiliando na compreensão dos aspectos estruturais e funcionais das comunidades, além de servirem como base para estudos sobre biodiversidade, biogeografia e evolução (Bertini, Fransozo, \& Melo, 2004, Mantelatto, Faria, Biagi, \& Melo, 2004, Almeida, Guerrazzi, \& Coelho, 2007, Coelho, Almeida, Bezerra, \& Souza-Filho, 2007, Almeida \& Coelho, 2008, Alves, Barros-Alves, Cobo, Lima, \& Fransozo, 2012, Pachelle, Anker, Mendes, \& Bezerra, 2016, Lima \& Martinelli-Lemos, 2019).

No Brasil, diversos estudos de levantamento taxonômico de crustáceos foram realizados, principalmente considerando os limites geográficos estaduais, áreas com importante diferencial ambiental ou grupos taxonômicos específicos (D’Incao, 1995a, Rieger, 1997, Fransozo, Costa, Mantelatto, Pinheiro, \& Santos, 2002, Bertini, Fransozo, \& Melo, 2004, Mantelatto et al., 2004, 2020, Coelho, Almeida, Souza-Filho, Bezerra, \& Giraldes, 2006, Coelho et al., 2007, Coelho, Almeida, \& Bezerra, 2008, Alves, Cobo, \& Melo, 2006, Alves, Ramos-Porto, Viana, 2008, Alves et al., 2012, Almeida, Guerrazzi, \& Coelho, 2007, Almeida, Souza, Boehs, \& Bezerra, 2010, Almeida et al., 2013, 2018, Almeida \& Coelho, 2008, Mossolin, Pileggi, \& Mantelatto, 2016, Boos et al., 2012, Anker, Tavares, \& Mendonça, 2016, Pachelle et al., 2016, Terossi et al., 2018, Lima \& Martinelli-Lemos, 2019). Devido à esforços de diferentes grupos de pesquisadores, possivelmente o litoral de São Paulo seja o mais estudado do Brasil no que se refere à fauna de crustáceos decápodes (Mantelatto et al. 2017, 2020, Terossi et al., 2018). Embora exista muita informação sobre a carcinofauna do estado, ainda existe uma carência de estudos de levantamento taxonômico de crustáceos na região do Complexo baía-estuário de Santos-São Vicente e canal de Bertioga (CBESSB).

Apesar da relevância geoeconômica da Baía de Santos, ainda são incipientes os estudos que abordem a composição taxonômica de sua carcinofauna. Assim, este estudo teve como objetivo fornecer informações sobre a composição, abundância e importância econômica da fauna de crustáceos da Baía de Santos, também apresentando uma lista copilada da carcinofauna do CBESSB, área fortemente impactada, localizada no litoral centro do estado de São Paulo.

\section{Metodologia}

\section{Caracterização da área}

A Baía de Santos é semi-abrigada e possui uma área de aproximadamente $43 \mathrm{~km}^{2}$, sua maior largura (cerca de $8,9 \mathrm{~km}$ ) se estende entre a Ponta do Itaipú em Praia Grande e a Ponta do Monduba no Guarujá, a região central possui largura com cerca de 7,4 km, entre a Praia de Itaquitanduva em São Vicente e a Ilha das Palmas no Guarujá, e a porção interna possui cerca de 6,3 km de largura (entre a ponta da Ilha Porchat em São Vicente e o deck do Pescador em Santos). No lado leste da Baía de Santos ocorre a dragagem para a entrada de navios no Porto de Santos, em contrapartida, o lado oeste, desde 1993, abriga o parque estadual do Xixová-Japuí, com área marinha de 3 km² (Santos, 2020).

O CBESSB abriga o maior porto marítimo da América Latina e um dos maiores polos petroquímicos do Brasil (Cubatão), sendo estes responsáveis por inúmeros impactos antropogênicos (Lamparelli et al. 2001, Medeiros \& Bícego, 2004, Martins, Gomes, Ferreira, \& Montone, 2008, Siqueira et al., 2012, Nunes et al., 2019). Além destes estressores, através do emissário submarino que funciona desde 1979 e possui uma vazão de aproximadamente $7.000 \mathrm{l} / \mathrm{s}$, os efluentes domésticos são lançados no interior da Baía de Santos (cerca de $4 \mathrm{~km}$ da costa) (CETESB, 2007). É um ambiente heterogêneo formado por praias arenosas, costões rochosos, manguezais e fundo lodoso (predominantemente argila e silte), apresentando sedimento em suspensão e areia na região do emissário submarino (Mandaji, 2008). Recebe águas provenientes do sistema Alto Tietê que penetram no seu interior de forma intermitente, por meio do canal de fuga da Usina Hidroelétrica de Henry Borden (CETESB, 2007). As águas da plataforma adjacente ao complexo estuarino de Santos-São Vicente entram no estuário através das marés, 
pela Baía de Santos e pelo Canal de Bertioga. No geral, as marés são semidiurnas de altura reduzida, variando entre 0,7 e 1,5 metro (quadratura e sizígia) (Castro, 2008).

\section{Monitoramento}

Foram realizadas quatro campanhas amostrais distribuídas trimestralmente (ciclos climáticos) entre abril e dezembro de 2017. As coletas foram efetuadas com a utilização de embarcação do tipo bote camaroeiro com tangones (com 10,5 m de comprimento e motor diesel de $60 \mathrm{hp}$ ) equipado com duas redes de arrasto de fundo ( $2 \mathrm{~m}$ de altura, $9 \mathrm{~m}$ de largura, malha de 20 mm entre nós opostos nas mangas/ corpo e $16 \mathrm{~mm}$ no ensacador), e duas portas com $120 \mathrm{~cm}$ de altura e $55 \mathrm{~cm}$ de largura (35 kg cada). Em cada campanha foram realizados seis arrastos de 30 minutos com velocidade aproximada de 1,5 nós, sendo três situados em alinhamento perpendicular ao canal do estuário de Santos (P2, P4 e P6) e três ao canal do estuário de São Vicente (P1, P3 e P5), equidistantes a aproximadamente $2.000 \mathrm{~m}$, possibilitando uma abrangência de toda a Baía de Santos (Figura 1). A distância média percorrida por ponto foi de aproximadamente $1.200 \mathrm{~m}$ (Tabela 1), totalizando $129.600 \mathrm{~m}^{2}$ de área varrida por campanha.

Com base nos pontos amostrais foram definidas três faixas de profundidade, sendo a primeira (P1 e P2) denominada

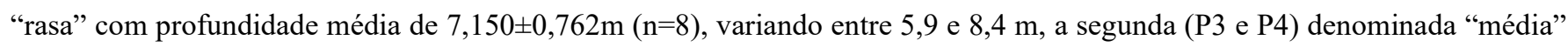
com $11,125 \pm 1,188 \mathrm{~m},(\mathrm{n}=8)$, variando entre 10,1 e 13,2 m, e a denominada "fundo" com 12,875 $\pm 1,512 \mathrm{~m}$ ( $\mathrm{n}=8)$, variando entre 11,3 e 16,1 m. Assim como adotado para as faixas batimétricas, os dados também foram organizados por posicionamento na Baía de Santos, sendo o lado de São Vicente composto pelos pontos P1, P3 e P5, e o lado de Santos por P2, P4 e P6.

Figura 1. Mapa do Complexo Baía estuário de Santos-São Vicente e canal de Bertioga, com a localização dos pontos amostrais na Baía de Santos.

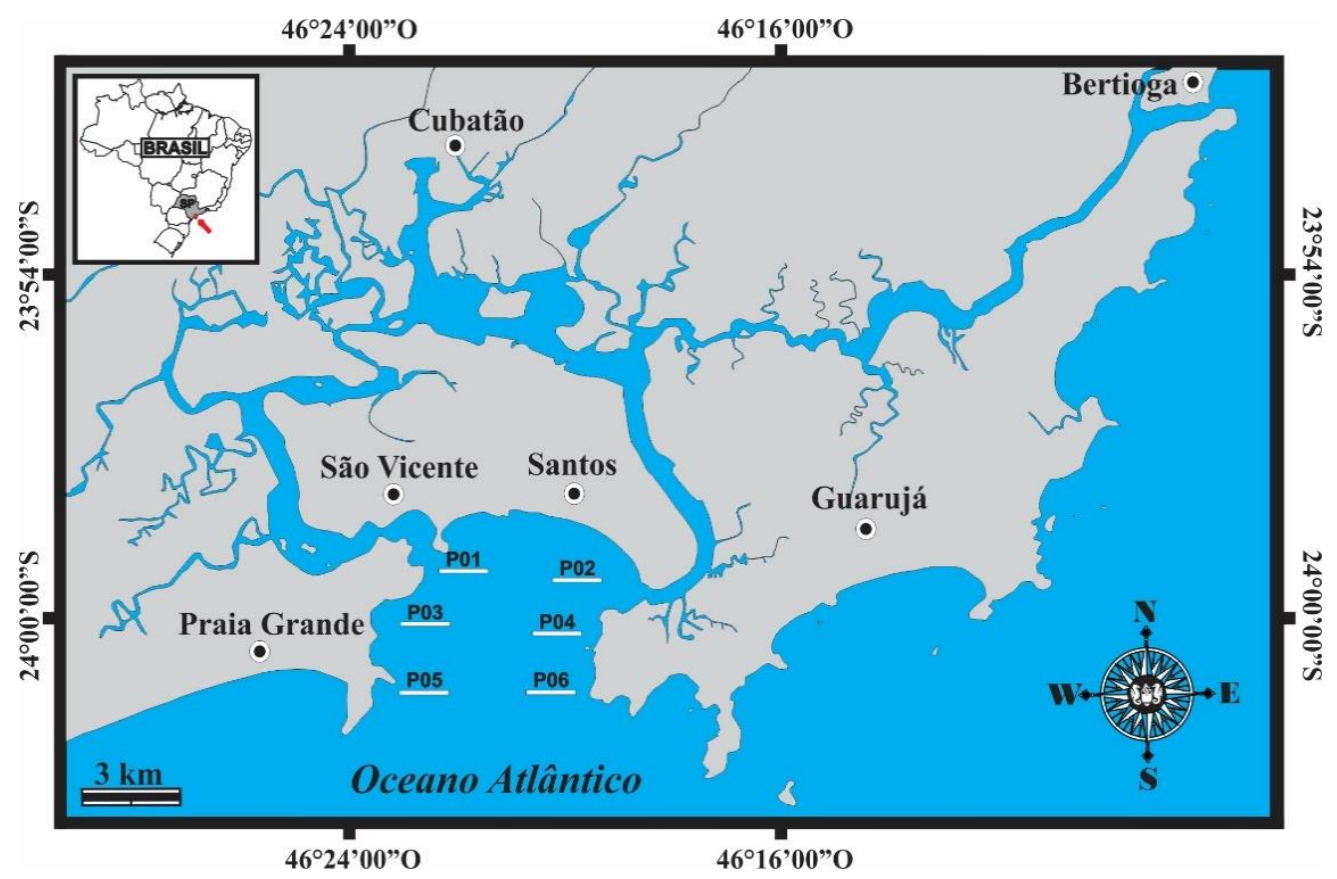

Fonte: Autores. 
Tabela 1. Coordenadas geográficas dos pontos amostrais localizados na Baía de Santos - SP, Brasil. (Lat $=$ latitude, Lon $=$ longitude, $\mathrm{i}=$ início, $\mathrm{f}=$ fim, $\mathrm{S}=$ Sul e $\mathrm{W}=$ Oeste).

\begin{tabular}{lcccccc}
\hline & Ponto 01 & Ponto 02 & Ponto 03 & Ponto 04 & Ponto 05 & Ponto 06 \\
\hline Lat (i) & $23^{\circ} 59^{\prime} 03^{\prime \prime S}$ & $23^{\circ} 59^{\prime} 30^{\prime \prime} \mathrm{S}$ & $24^{\circ} 00^{\prime} 06^{\prime \prime S}$ & $24^{\circ} 00^{\prime} 31^{\prime \prime S}$ & $24^{\circ} 01^{\prime} 22^{\prime \prime S}$ & $24^{\circ} 01^{\prime} 31^{\prime \prime S}$ \\
Lon (i) & $46^{\circ} 22^{\prime} 01^{\prime \prime} \mathrm{W}$ & $46^{\circ} 19^{\prime} 14^{\prime \prime} \mathrm{W}$ & $46^{\circ} 22^{\prime} 55^{\prime \prime} \mathrm{W}$ & $46^{\circ} 19^{\prime} 43^{\prime \prime} \mathrm{W}$ & $46^{\circ} 22^{\prime} 51^{\prime \prime} \mathrm{W}$ & $46^{\circ} 19^{\prime} 55^{\prime \prime} \mathrm{W}$ \\
Lat (f) & $23^{\circ} 59^{\prime} 15^{\prime \prime S}$ & $23^{\circ} 59^{\prime} 20^{\prime \prime S}$ & $24^{\circ} 00^{\prime} 19^{\prime \prime} \mathrm{S}$ & $24^{\circ} 00^{\prime} 21^{\prime \prime S}$ & $24^{\circ} 01^{\prime} 34^{\prime \prime} \mathrm{S}$ & $24^{\circ} 01^{\prime} 20^{\prime \prime} \mathrm{S}$ \\
Lon (f) & $46^{\circ} 21^{\prime} 19^{\prime \prime} \mathrm{W}$ & $46^{\circ} 19^{\prime} 57^{\prime \prime} \mathrm{W}$ & $46^{\circ} 22^{\prime} 15^{\prime \prime} \mathrm{W}$ & $46^{\circ} 20^{\prime} 25^{\prime \prime} \mathrm{W}$ & $46^{\circ} 22^{\prime} 12^{\prime \prime} \mathrm{W}$ & $46^{\circ} 20^{\prime} 38^{\prime \prime} \mathrm{W}$ \\
\hline
\end{tabular}

Fonte: Autores.

Em laboratório, os exemplares de crustáceos foram quantificados e pesados após a identificação realizada com base em D’Incao (1995b) e Pérez Farfante e Kensley (1997) para Penaeidae, Holthuis (1993), Costa, Fransozo, Melo e Freire (2003), Guterres (2003), Rhyne e Lin (2006) e Ferreira, Vieira e D'incao (2010) para Caridea, Melo (1996) para Brachyura, Melo (1999) para Anomura. A nomenclatura científica foi verificada junto ao World Register of Marine Species (WORMS, 2020).

As espécies também foram caracterizadas quanto a importância econômica para alimentação humana, utilização como isca para pesca esportiva e ornamentação (aquariofilia).

\section{Resultados}

\subsection{Monitoramento}

Foram capturados um total de 13.404 indivíduos que totalizaram 91.890,07 g, pertencentes a 18 espécies, 15 gêneros, 11 Famílias, quatro infraordens e uma ordem (Decapoda) (Tabela 2).

A infraordem com maior representatividade de famílias foi Brachyura (36,4\%), seguida de Anomura (27,3\%) e Dendrobranchiata $(27,3 \%)$, que juntas representaram 90,9\% do total de espécies capturadas (Figura 2).

Figura 2. Número de famílias por infraordens de crustáceos registrados no monitoramento realizado na Baía de Santos entre abril e dezembro de 2017.

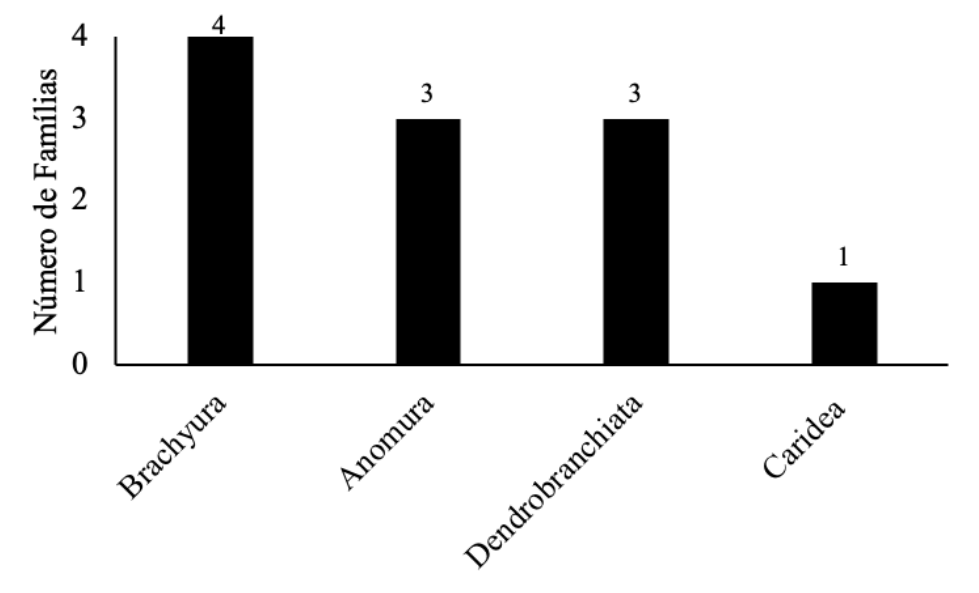

Fonte: Autores. 
A infraordem com maior número de espécies foi Brachyura (44,4\%), seguida de Dendrobranchiata $(33,3 \%)$. Estas, representaram 77,8\% do número total de espécies capturadas (Figura 3).

A família com maior representatividade de espécies foi Portunidae (27,8\%), seguida de Penaeidae (22,2\%), que juntas representaram $50 \%$ do total capturado (Figura 4 ).

Figura 3. Número de espécies por infraordens de crustáceos registrados no monitoramento realizado na Baía de Santos entre abril e dezembro de 2017.

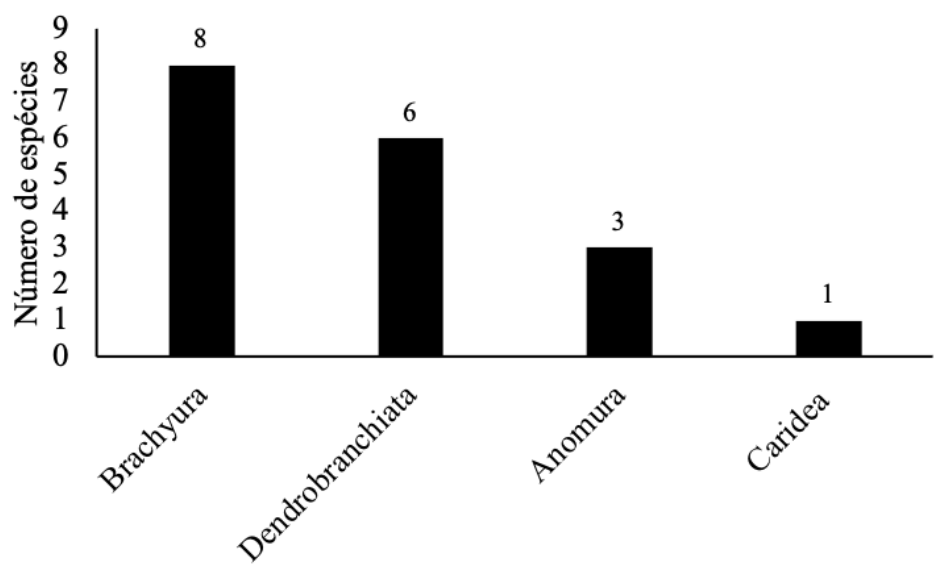

Fonte: Autores.

Figura 4. Número de espécies por famílias de crustáceos registrados no monitoramento realizado na Baía de Santos entre abril e dezembro de 2017

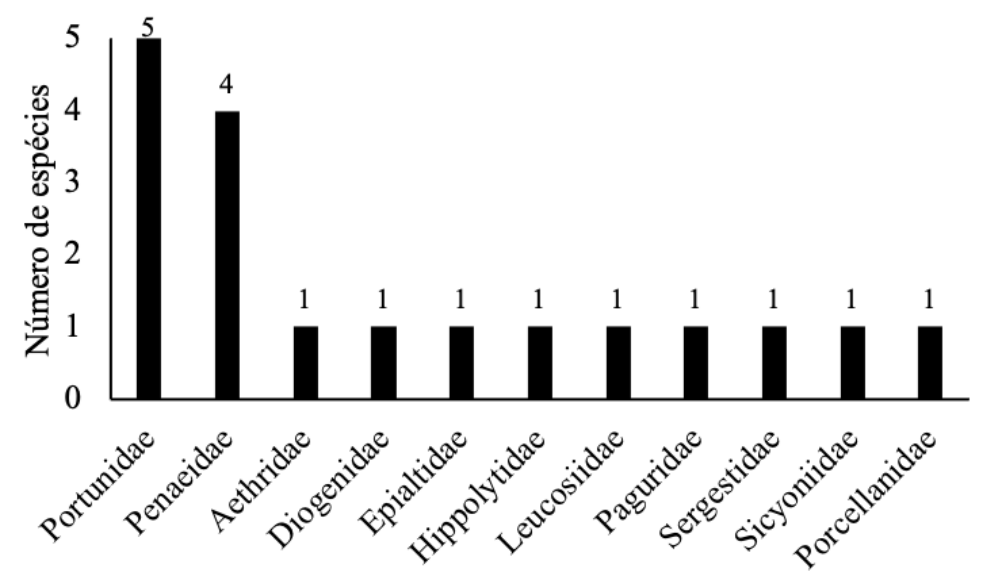

Fonte: Autores. 


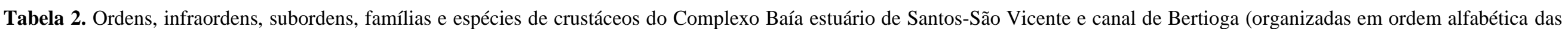

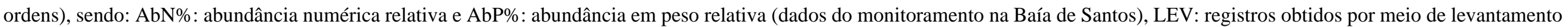
bibliográfico, R\$: importância econômica, O: ornamentação (aquariofilia), A: alimentação e isca para pesca.

\begin{tabular}{|c|c|c|c|c|c|c|c|}
\hline Ordem & Infraordem/Subordem & Família & Lista espécies & $\mathrm{AbN} \%$ & $\mathrm{AbP} \%$ & LEV & $\mathrm{R} \$$ \\
\hline Decapoda & Anomura & Diogenidae & Clibanarius vittatus (Bosc, 1801) & 0,05 & 0,09 & $\mathrm{x}$ & $\mathrm{O}$ \\
\hline Decapoda & Anomura & Diogenidae & Dardanus insignis (de Saussure, 1857) & 0 & 0 & $\mathrm{x}$ & $\mathrm{O}$ \\
\hline Decapoda & Anomura & Diogenidae & Isocheles sawayai Forest \& de Saint Laurent, 1968 & 0 & 0 & $\mathrm{x}$ & $\mathrm{O}$ \\
\hline Decapoda & Anomura & Diogenidae & Loxopagurus loxochelis (Moreira, 1901) & 0 & 0 & $\mathrm{x}$ & $\mathrm{O}$ \\
\hline Decapoda & Anomura & Diogenidae & Paguristes tortugae Schmitt, 1933 & 0 & 0 & $\mathrm{x}$ & $\mathrm{O}$ \\
\hline Decapoda & Anomura & Diogenidae & Petrochirus diogenes (Linnaeus, 1758) & 0 & 0 & $\mathrm{x}$ & $\mathrm{O}$ \\
\hline Decapoda & Anomura & Paguridae & Pagurus criniticornis (Dana, 1852) & 0 & 0 & $\mathrm{x}$ & $\mathrm{O}$ \\
\hline Decapoda & Anomura & Paguridae & Pagurus longicarpus Say, 1817 & 0,01 & 0,46 & - & $\mathrm{O}$ \\
\hline Decapoda & Anomura & Porcellanidae & Megalobrachium roseum (Rathbun, 1900) & 0 & 0 & $\mathrm{x}$ & - \\
\hline Decapoda & Anomura & Porcellanidae & Pachycheles laevidactylus Ortmann, 1892 & 0 & 0 & $\mathrm{x}$ & - \\
\hline Decapoda & Anomura & Porcellanidae & Pachycheles monilifer (Dana, 1852) & 0 & 0 & $\mathrm{x}$ & - \\
\hline Decapoda & Anomura & Porcellanidae & Petrolisthes armatus (Gibbes, 1850) & 0 & 0 & $\mathrm{x}$ & - \\
\hline Decapoda & Anomura & Porcellanidae & Pisidia brasiliensis Rodrigues da Costa, 1968 & 0,01 & 0,01 & $\mathrm{x}$ & - \\
\hline Decapoda & Axiidea & Callichiridae & Callichirus major (Say, 1818) & 0 & 0 & $\mathrm{x}$ & A \\
\hline Decapoda & Brachyura & Aethridae & Hepatus pudibundus (Herbst, 1785) & 0,19 & 1,16 & $\mathrm{x}$ & - \\
\hline Decapoda & Brachyura & Epialtidae & Apiomithrax violaceus (Milne-Edwards, 1867) & 0 & 0 & $\mathrm{x}$ & - \\
\hline Decapoda & Brachyura & Epialtidae & Libinia ferreirae de Brito Capello, 1871 & 0 & 0 & $\mathrm{x}$ & - \\
\hline Decapoda & Brachyura & Epialtidae & Libinia spinosa Guérin, 1832 & 0,18 & 0,26 & - & - \\
\hline Decapoda & Brachyura & Epialtidae & Notolopas brasiliensis Miers, 1886 & 0 & 0 & $\mathrm{x}$ & - \\
\hline Decapoda & Brachyura & Eriphiidae & Eriphia gonagra (Fabricius, 1781) & 0 & 0 & $\mathrm{x}$ & - \\
\hline Decapoda & Brachyura & Euryplacidae & Euryplax nitida Stimpson, 1859 & 0 & 0 & $\mathrm{x}$ & - \\
\hline Decapoda & Brachyura & Gecarcinidae & Cardisoma guanhumi Latreille, Le Peletier, Serville \& Guérin, 1828 & 0 & 0 & $\mathrm{x}$ & A \\
\hline Decapoda & Brachyura & Grapsidae & Goniopsis cruentata (Latreille, 1803) & 0 & 0 & $\mathrm{x}$ & $\mathrm{A}$ \\
\hline Decapoda & Brachyura & Grapsidae & Pachygrapsus gracilis (de Saussure, 1857) & 0 & 0 & $\mathrm{x}$ & - \\
\hline Decapoda & Brachyura & Grapsidae & Pachygrapsus transversus (Gibbes, 1850) & 0 & 0 & $\mathrm{x}$ & A \\
\hline Decapoda & Brachyura & Inachidae & Stenorhynchus seticornis (Herbst, 1788) & 0 & 0 & $\mathrm{x}$ & - \\
\hline Decapoda & Brachyura & Leucosiidae & Persephona mediterranea (Herbst, 1794) & 0 & 0 & $\mathrm{x}$ & $\mathrm{O}$ \\
\hline Decapoda & Brachyura & Leucosiidae & Persephona punctata (Linnaeus, 1758) & 0,2 & 0,15 & $\mathrm{x}$ & $\mathrm{O}$ \\
\hline
\end{tabular}


Research, Society and Development, v. 10, n. 1, e17210110682, 2021

(CC BY 4.0) | ISSN 2525-3409 | DOI: http://dx.doi.org/10.33448/rsd-v10i1.10682

\begin{tabular}{|c|c|c|c|c|c|c|c|}
\hline Ordem & Infraordem/Subordem & Família & Lista espécies & $\mathrm{AbN} \%$ & $\mathrm{AbP} \%$ & LEV & $\mathrm{R} \$$ \\
\hline Decapoda & Brachyura & Menippidae & Menippe nodifrons Stimpson, 1859 & 0 & 0 & $\mathrm{x}$ & A \\
\hline Decapoda & Brachyura & Mithracidae & Omalacantha bicornuta (Latreille, 1825) & 0 & 0 & $\mathrm{x}$ & - \\
\hline Decapoda & Brachyura & Ocypodidae & Leptuca leptodactyla (Rathbun, in Rankin, 1898) & 0 & 0 & $\mathrm{x}$ & - \\
\hline Decapoda & Brachyura & Ocypodidae & Leptuca uruguayensis (Nobili, 1901) & 0 & 0 & $\mathrm{x}$ & - \\
\hline Decapoda & Brachyura & Ocypodidae & Minuca vocator (Herbst, 1804) & 0 & 0 & $\mathrm{x}$ & - \\
\hline Decapoda & Brachyura & Ocypodidae & Ucides cordatus (Linnaeus, 1763) & 0 & 0 & $\mathrm{x}$ & $\mathrm{O}$ \\
\hline Decapoda & Brachyura & Panopeidae & Acantholobulus schmitti (Rathbun, 1930) & 0 & 0 & $\mathrm{x}$ & - \\
\hline Decapoda & Brachyura & Panopeidae & Eurypanopeus abbreviatus (Stimpson, 1860) & 0 & 0 & $\mathrm{x}$ & - \\
\hline Decapoda & Brachyura & Panopeidae & Eurytium limosum (Say, 1818) & 0 & 0 & $\mathrm{x}$ & - \\
\hline Decapoda & Brachyura & Panopeidae & Hexapanopeus angustifrons (Benedict \& Rathbun, 1891) & 0 & 0 & $\mathrm{x}$ & - \\
\hline Decapoda & Brachyura & Panopeidae & Hexapanopeus paulensis Rathbun, 1930 & 0 & 0 & $\mathrm{x}$ & - \\
\hline Decapoda & Brachyura & Panopeidae & Panopeus americanus de Saussure, 1857 & 0 & 0 & $\mathrm{x}$ & - \\
\hline Decapoda & Brachyura & Panopeidae & Panopeus austrobesus Williams, 1983 & 0 & 0 & $\mathrm{x}$ & - \\
\hline Decapoda & Brachyura & Panopeidae & Panopeus lacustris Desbonne in Desbonne \& Schramm, 1867 & 0 & 0 & $\mathrm{x}$ & - \\
\hline Decapoda & Brachyura & Panopeidae & Panopeus rugosus Milne-Edwards, 1880 & 0 & 0 & $\mathrm{x}$ & - \\
\hline Decapoda & Brachyura & Parthenopidae & Costalambrus tommasii (Rodrigues da Costa, 1959) & 0 & 0 & $\mathrm{x}$ & - \\
\hline Decapoda & Brachyura & Parthenopidae & Heterocrypta granulata (Gibbes, 1850) & 0 & 0 & $\mathrm{x}$ & - \\
\hline Decapoda & Brachyura & Parthenopidae & Heterocrypta lapidea Rathbun, 1901 & 0 & 0 & $\mathrm{x}$ & - \\
\hline Decapoda & Brachyura & Parthenopidae & Mesorhoea sexspinosa Stimpson, 1871 & 0 & 0 & $\mathrm{x}$ & - \\
\hline Decapoda & Brachyura & Pilumnidae & Pilumnus caribaeus Desbonne \& Schramm, 1867 & 0 & 0 & $\mathrm{x}$ & - \\
\hline Decapoda & Brachyura & Portunidae & Achelous spinicarpus Stimpson, 1871 & 0 & 0 & $\mathrm{x}$ & - \\
\hline Decapoda & Brachyura & Portunidae & Achelous spinimanus (Latreille, 1819) & 0,1 & 0,21 & $\mathrm{x}$ & A \\
\hline Decapoda & Brachyura & Portunidae & Arenaeus cribrarius (Lamarck, 1818) & 0,11 & 0,21 & $\mathrm{x}$ & A \\
\hline Decapoda & Brachyura & Portunidae & Callinectes bocourti Milne-Edwards, 1879 & 0 & 0 & $\mathrm{x}$ & A \\
\hline Decapoda & Brachyura & Portunidae & Callinectes danae Smith, 1869 & 4,45 & 27,69 & $\mathrm{x}$ & A \\
\hline Decapoda & Brachyura & Portunidae & Callinectes exasperatus (Gerstaecker, 1856) & 0 & 0 & $\mathrm{x}$ & A \\
\hline Decapoda & Brachyura & Portunidae & Callinectes marginatus (Milne-Edwards, 1861) & 0 & 0 & $\mathrm{x}$ & A \\
\hline Decapoda & Brachyura & Portunidae & Callinectes ornatus Ordway, 1863 & 4,3 & 9,17 & $\mathrm{x}$ & A \\
\hline Decapoda & Brachyura & Portunidae & Callinectes sapidus Rathbun, 1896 & 0,06 & 0,77 & $\mathrm{x}$ & A \\
\hline Decapoda & Brachyura & Portunidae & Charybdis hellerii (Milne-Edwards, 1867) & 0 & 0 & $\mathrm{x}$ & - \\
\hline Decapoda & Brachyura & Portunidae & Charybdis variegata (Fabricius, 1798) & 0 & 0 & $\mathrm{x}$ & - \\
\hline Decapoda & Brachyura & Portunidae & Cronius ruber (Lamarck, 1818) & 0 & 0 & $\mathrm{x}$ & - \\
\hline
\end{tabular}


Research, Society and Development, v. 10, n. 1, e17210110682, 2021

(CC BY 4.0) | ISSN 2525-3409 | DOI: http://dx.doi.org/10.33448/rsd-v10i1.10682

\begin{tabular}{|c|c|c|c|c|c|c|c|}
\hline Ordem & Infraordem/Subordem & Família & Lista espécies & $\mathrm{AbN} \%$ & $\mathrm{AbP} \%$ & LEV & $\mathrm{R} \$$ \\
\hline Decapoda & Brachyura & Sesarmidae & Aratus pisonii (Milne Edwards, 1837 & 0 & 0 & $\mathrm{x}$ & A \\
\hline Decapoda & Brachyura & Sesarmidae & Armases rubripes (Rathbun, 1897) & 0 & 0 & $\mathrm{x}$ & - \\
\hline Decapoda & Brachyura & Sesarmidae & Sesarma rectum Randall, 1840 & 0 & 0 & $\mathrm{x}$ & - \\
\hline Decapoda & Brachyura & Trichodactylidae & Trichodactylus dentatus Milne Edwards, 1853 & 0 & 0 & $\mathrm{x}$ & - \\
\hline Decapoda & Brachyura & Trichodactylidae & Trichodactylus panoplus (Von Martens, 1869) & 0 & 0 & $\mathrm{x}$ & - \\
\hline Decapoda & Brachyura & Trichodactylidae & Trichodactylus petropolitanus (Göldi, 1886) & 0 & 0 & $\mathrm{x}$ & - \\
\hline Decapoda & Brachyura & Trichodactylidae & Trichodactylus fluviatilis Latreille, 1828 & 0 & 0 & $\mathrm{x}$ & A \\
\hline Decapoda & Caridea & Alpheidae & Alpheus armillatus Milne Edwards, 1837 & 0 & 0 & $\mathrm{x}$ & - \\
\hline Decapoda & Caridea & Alpheidae & Alpheus formosus Gibbes, 1850 & 0 & 0 & $\mathrm{x}$ & - \\
\hline Decapoda & Caridea & Alpheidae & Alpheus intrinsecus Spence Bate, 1888 & 0 & 0 & $\mathrm{x}$ & - \\
\hline Decapoda & Caridea & Alpheidae & Alpheus lobidens De Haan, 1849 & 0 & 0 & $\mathrm{x}$ & - \\
\hline Decapoda & Caridea & Alpheidae & Alpheus pontederiae de Rochebrune, 1883 & 0 & 0 & $\mathrm{x}$ & - \\
\hline Decapoda & Caridea & Alpheidae & Athanas nitescens (Leach, 1814) & 0 & 0 & $\mathrm{x}$ & - \\
\hline Decapoda & Caridea & Alpheidae & Synalpheus apioceros Coutière, 1909 & 0 & 0 & $\mathrm{x}$ & - \\
\hline Decapoda & Caridea & Hippolytidae & Exhippolysmata oplophoroides (Holthuis, 1948) & 0,07 & 0 & $\mathrm{x}$ & - \\
\hline Decapoda & Caridea & Lysmatidae & Lysmata ankeri Rhyne \& Lin, 2006 & 0 & 0 & $\mathrm{x}$ & $\mathrm{O}$ \\
\hline Decapoda & Caridea & Lysmatidae & Lysmata rauli Laubenheimer \& Rhyne, 2010 & 0 & 0 & $\mathrm{x}$ & $\mathrm{O}$ \\
\hline Decapoda & Caridea & Lysmatidae & Lysmata vittata (Stimpson, 1860) & 0 & 0 & $\mathrm{x}$ & $\mathrm{O}$ \\
\hline Decapoda & Caridea & Palaemonidae & Leander paulensis Ortmann, 1897 & 0 & 0 & $\mathrm{x}$ & - \\
\hline Decapoda & Caridea & Palaemonidae & Leander potitinga Müller, 1892 & 0 & 0 & $\mathrm{x}$ & - \\
\hline Decapoda & Caridea & Palaemonidae & Macrobrachium acanthurus (Wiegmann, 1836) & 0 & 0 & $\mathrm{x}$ & $\mathrm{AO}$ \\
\hline Decapoda & Caridea & Palaemonidae & Macrobrachium carcinus (Linnaeus, 1758) & 0 & 0 & $\mathrm{x}$ & $\mathrm{AO}$ \\
\hline Decapoda & Caridea & Palaemonidae & Macrobrachium nattereri (Heller, 1862) & 0 & 0 & $\mathrm{x}$ & $\mathrm{AO}$ \\
\hline Decapoda & Caridea & Palaemonidae & Macrobrachium olfersii (Wiegmann, 1836) & 0 & 0 & $\mathrm{x}$ & $\mathrm{AO}$ \\
\hline Decapoda & Caridea & Palaemonidae & Macrobrachium vollenhoveni (Herklots, 1857) & 0 & 0 & $\mathrm{x}$ & $\mathrm{AO}$ \\
\hline Decapoda & Caridea & Palaemonidae & Nematopalaemon schmitti (Holthuis, 1950) & 0 & 0 & $\mathrm{x}$ & $\mathrm{AO}$ \\
\hline Decapoda & Caridea & Palaemonidae & Palaemon iheringi Ortmann, 1897 & 0 & 0 & $\mathrm{x}$ & - \\
\hline Decapoda & Caridea & Palaemonidae & Palaemon olfersii Wiegmann, 1836 & 0 & 0 & $\mathrm{x}$ & - \\
\hline Decapoda & Caridea & Palaemonidae & Palaemon paivai Fausto Filho, 1967 & 0 & 0 & $\mathrm{x}$ & - \\
\hline Decapoda & Caridea & Palaemonidae & Periclimenes paivai Chace, 1969 & 0 & 0 & $\mathrm{x}$ & - \\
\hline Decapoda & Dendrobranchiata & Penaeidae & Artemesia longinaris Spence Bate, 1888 & 0,01 & 0,02 & $\mathrm{x}$ & A \\
\hline Decapoda & Dendrobranchiata & Penaeidae & Penaeus brasiliensis Latreille, 1817 & 0,04 & 0,26 & $\mathrm{x}$ & A \\
\hline
\end{tabular}


Research, Society and Development, v. 10, n. 1, e17210110682, 2021

(CC BY 4.0) | ISSN 2525-3409 | DOI: http://dx.doi.org/10.33448/rsd-v10i1.10682

\begin{tabular}{|c|c|c|c|c|c|c|c|}
\hline Ordem & Infraordem/Subordem & Família & Lista espécies & $\mathrm{AbN} \%$ & $\mathrm{AbP} \%$ & LEV & $\mathrm{R} \$$ \\
\hline Decapoda & Dendrobranchiata & Penaeidae & Penaeus paulensis (Pérez Farfante, 1967) & 0 & 0 & $\mathrm{x}$ & A \\
\hline Decapoda & Dendrobranchiata & Penaeidae & Penaeus setiferus (Linnaeus, 1767) & 0 & 0 & $\mathrm{x}$ & - \\
\hline Decapoda & Dendrobranchiata & Penaeidae & Penaeus schmitti Burkenroad, 1936 & 0,6 & 1,23 & $\mathrm{x}$ & A \\
\hline Decapoda & Dendrobranchiata & Penaeidae & Rimapenaeus constrictus (Stimpson, 1871) & 0 & 0 & $\mathrm{x}$ & - \\
\hline Decapoda & Dendrobranchiata & Penaeidae & Xiphopenaeus kroyeri (Heller, 1862) & 88,65 & 58,27 & $\mathrm{x}$ & A \\
\hline Decapoda & Dendrobranchiata & Sergestidae & Acetes americanus Ortmann, 1893 & 0,01 & 0 & $\mathrm{x}$ & - \\
\hline Decapoda & Dendrobranchiata & Sicyoniidae & Sicyonia dorsalis Kingsley, 1878 & 1,05 & 0,04 & $\mathrm{x}$ & - \\
\hline Decapoda & Dendrobranchiata & Solenoceridae & Pleoticus muelleri (Spence Bate, 1888) & 0 & 0 & $\mathrm{x}$ & A \\
\hline Isopoda & Oniscidea & Ligiidae & Ligia (Megaligia) exotica Roux, 1828 & 0 & 0 & $\mathrm{x}$ & - \\
\hline Sessilia & Balanomorpha & Balanidae & Amphibalanus eburneus (Gould, 1841) & 0 & 0 & $\mathrm{x}$ & - \\
\hline
\end{tabular}

Fonte: Autores e dados adaptados de Luederwaldt (1919), Tommasi (1967), Pita, Rodrigues, Graça Lopes e Coelho (1985), Moreira, Paiva Filho, Okida, Schmiegelow e Giannini (1988), Micheletti-Flores e Negreiros-Fransozo (1999), Reigada et al. (2006), Ferreira et al. (2010), Sant'Anna, Watanabe, Turra e Zara (2012), Simões (2012), Scalco et al. (2014), Souza (2017), Terossi et al. (2018), Costa, Deus e Rodrigues (2020). 
O gênero que apresentou o maior número de espécies foi Callinectes (16,7\%), seguido de Penaeus (11,1\%), que juntos representaram $27,8 \%$ de todas as espécies capturadas (Figura 5).

Figura 5. Número de espécies por gêneros de crustáceos registrados no monitoramento realizado na Baía de Santos entre abril e dezembro de 2017.

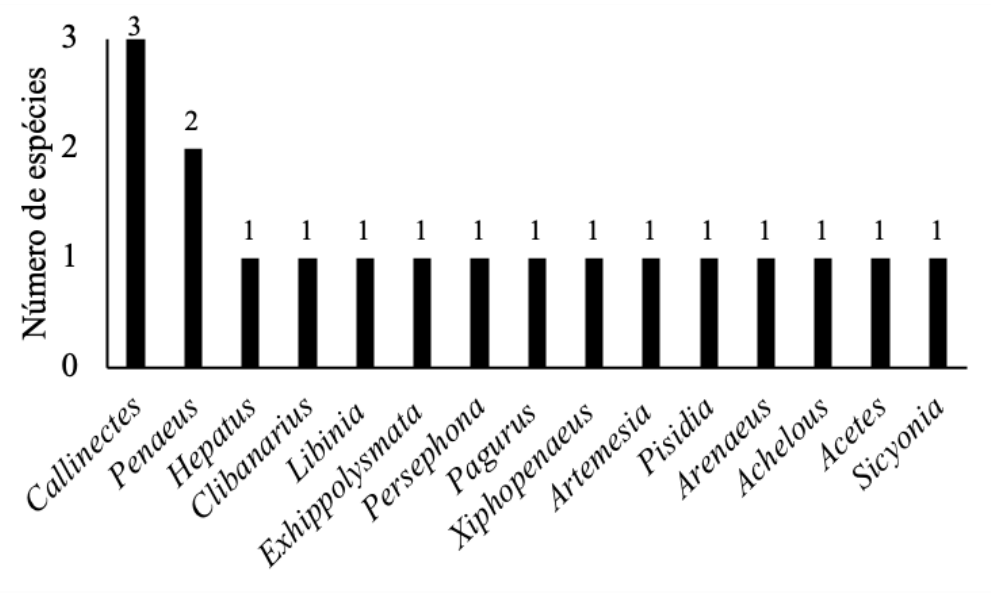

Fonte: Autores.

As espécies com a maior abundância numérica foram X. kroyeri com 11.882 exemplares (88,6\%), C. danae com 597 exemplares (4,5\%) e C. ornatus com 576 exemplares (4,3\%). Estas espécies também representaram a maior abundância em peso, sendo: X. kroyeri com 53.548,6 g (58,3\%), seguida de C. danae com 25.447,7 g (27,7\%) e C. ornatus com 8.424,9 g (9,17\%).

A média de captura para os seis pontos de coleta foi de 2.224,0 $\pm 1.420,67$ exemplares por ponto amostral ( $\mathrm{n}=6$ ), sendo P1 o ponto com o maior número de indivíduos capturados (4.159). Em relação a abundância em peso, a média foi de $15.315,0 \pm 6.479,85 \mathrm{~g}(\mathrm{n}=6)$ por ponto, com a maior abundância no Ponto P4.

Em média foram capturados 3.336 $\pm 2.761,4$ exemplares por estação climática $(n=4)$, sendo registrada a maior captura durante o inverno (7.156). Em relação a abundância em peso, a média capturada foi de 22.972,5 $\pm 11.820,50$ g (n=4) por período climático, sendo a maior abundância observada no inverno $(39.226,4 \mathrm{~g})$.

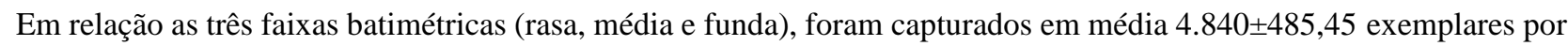
faixa $(n=3)$. Em relação a abundância em peso, a média de captura foi 30.630,02 $\pm 8.205,49 \mathrm{~g}(\mathrm{n}=3)$ por faixa de profundidade, com o maior valor na faixa média $(43.021,7 \mathrm{~g})$.

Considerando o posicionamento na Baía de Santos, observa-se que a média foi de 6.672 $\pm 5.208,5$ exemplares por posição $(\mathrm{n}=2)$, sendo o lado de São Vicente com maior representatividade (10.355). Em relação a abundância em peso, a média de captura

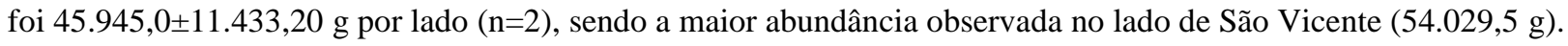

Com base na classificação sobre a importância econômica observa-se que $50 \%$ das espécies são utilizadas na alimentação humana e/ou para iscas na pesca esportiva, 16,67\% para aquariofilia e 33,33\% não possuem valor comercial. Considerando a abundância numérica e em peso (respectivamente), 98,3\% e 97,8\% são utilizadas na alimentação humana e/ou para iscas na pesca esportiva, $1,5 \%$ e $1,5 \%$ não possuem valor comercial e $0,2 \%$ e $0,7 \%$ são consideradas ornamentais.

\subsection{Levantamento bibliográfico}

Com base no levantamento bibliográfico, onde foram analisados 13 estudos (Luederwaldt, 1919, Tommasi, 1967, Pita et al., 1985, Moreira et al., 1988, Micheletti-Flores \& Negreiros-Fransozo, 1999, Reigada et al., 2006, Ferreira et al., 2010, 
Sant'Anna et al., 2012, Simões, 2012, Scalco et al. 2014, Souza, 2017, Terossi et al., 2018, Costa et al., 2020), foram registradas 100 espécies, pertencentes a 67 gêneros, 31 famílias, sete infraordens e três ordens (Tabela 2).

A ordem que apresentou o maior número de famílias foi Decapoda $(93,5 \%)$, seguida de Isopoda $(3,2 \%)$ e Sessilia $(3,2 \%)$ (Figura 6).

Figura 6. Número de famílias por ordens de crustáceos registrados no Complexo Baía estuário de Santos-São Vicente e canal de Bertioga através de levantamento bibliográfico.

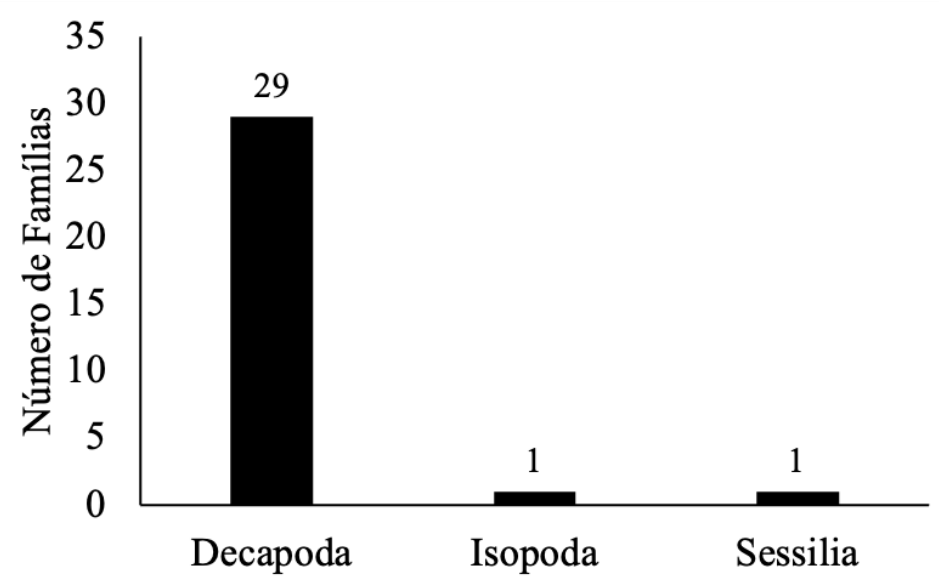

Fonte: Autores.

A infraordem com maior representatividade de famílias foi Brachyura (54,8\%), seguida de Caridea (12,9\%) e Dendrobranchiata (12,9\%), que juntas representaram 80,6\% do total registrado (Figura 7).

Figura 7. Número de famílias por infraordens de crustáceos registrados no Complexo Baía estuário de Santos-São Vicente e canal de Bertioga através de levantamento bibliográfico.

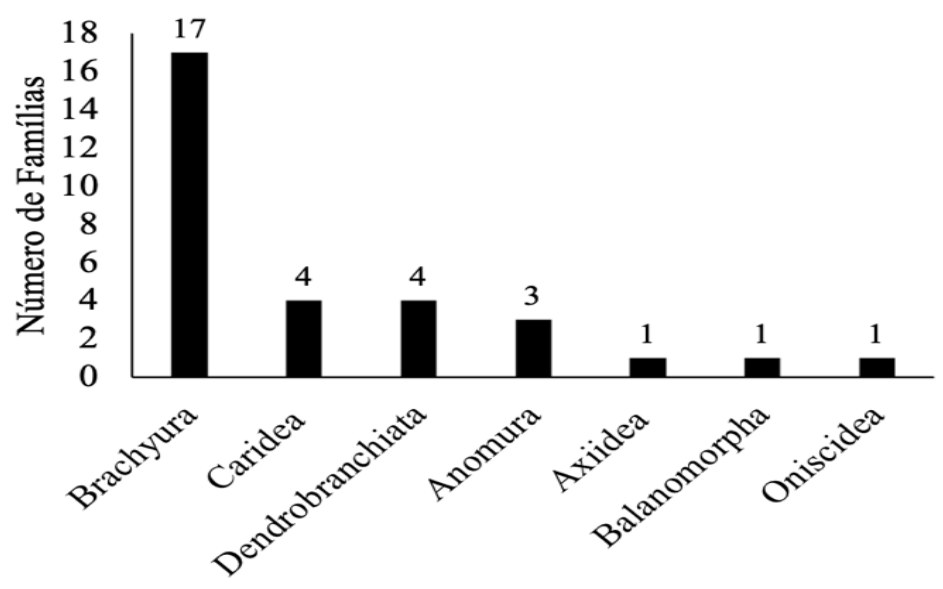

Fonte: Autores.

A infraordem com maior número de espécies foi Brachyura (52\%), seguida de Caridea (23\%), que juntas representaram $75 \%$ do total registrado (Figura 8). 
Figura 8. Número de espécies por infraordens de crustáceos registrados no Complexo Baía estuário de Santos-São Vicente e canal de Bertioga através de levantamento bibliográfico.

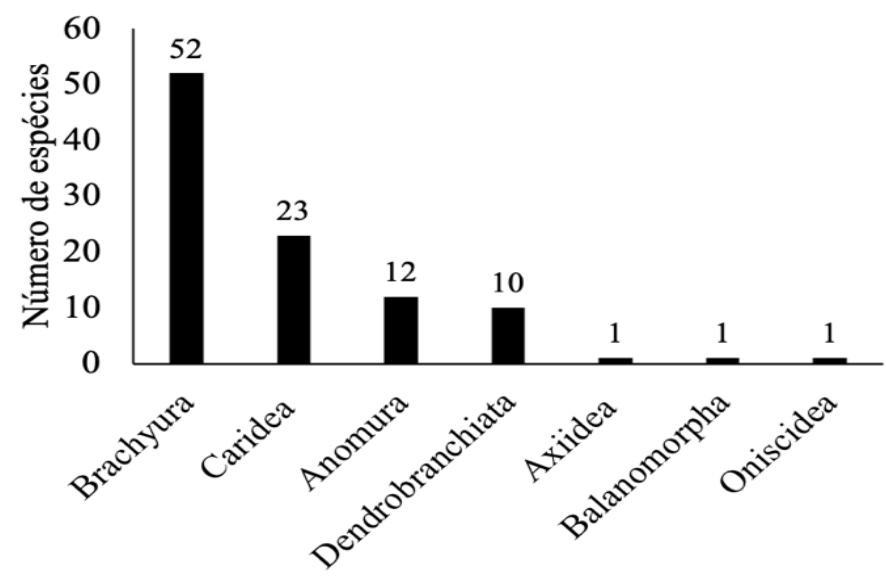

Fonte: Autores.

As famílias que apresentaram o maior número de espécies foram Palaemonida (12\%), Portunidae (12\%), seguidas de Panopeidae (9\%), Penaeidae (7\%) e Alpheidae (7\%), que juntas representaram 47\% do total de espécies registradas (Figura 9).

Figura 9. Número de espécies por famílias de crustáceos registrados no Complexo Baía estuário de Santos-São Vicente e canal de Bertioga através de levantamento bibliográfico.

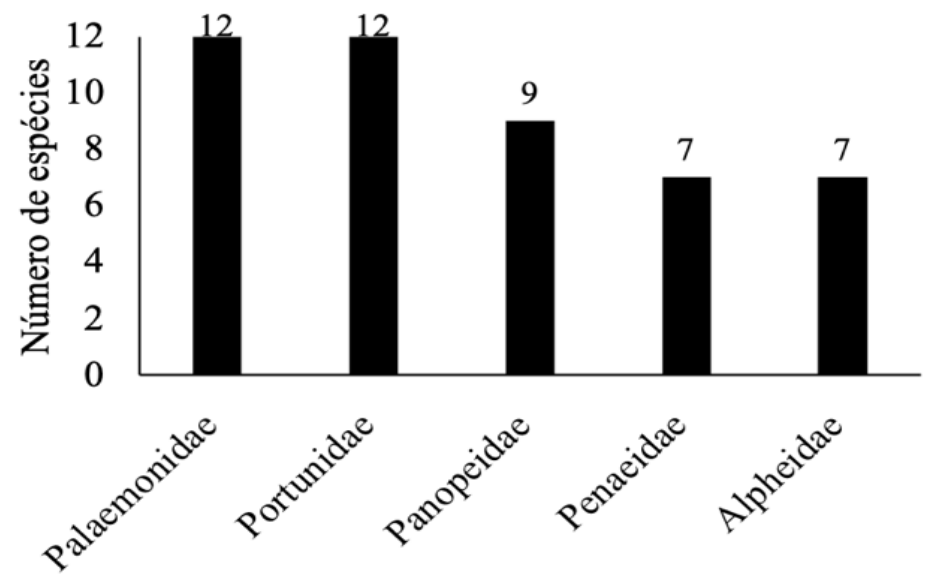

Fonte: Autores.

O gênero com maior representatividade de espécies foi Callinectes (6\%), seguido de Alpheus (5\%), Macrobrachium (5\%), Penaeus (4\%), Panopeus (4\%) e Trichodactylus (4\%), que juntos representaram $28 \%$ do total de espécies registradas (Figura 10). 
Figura 10. Número de espécies por gêneros de crustáceos registrados no Complexo Baía estuário de Santos-São Vicente e canal de Bertioga através de levantamento bibliográfico.

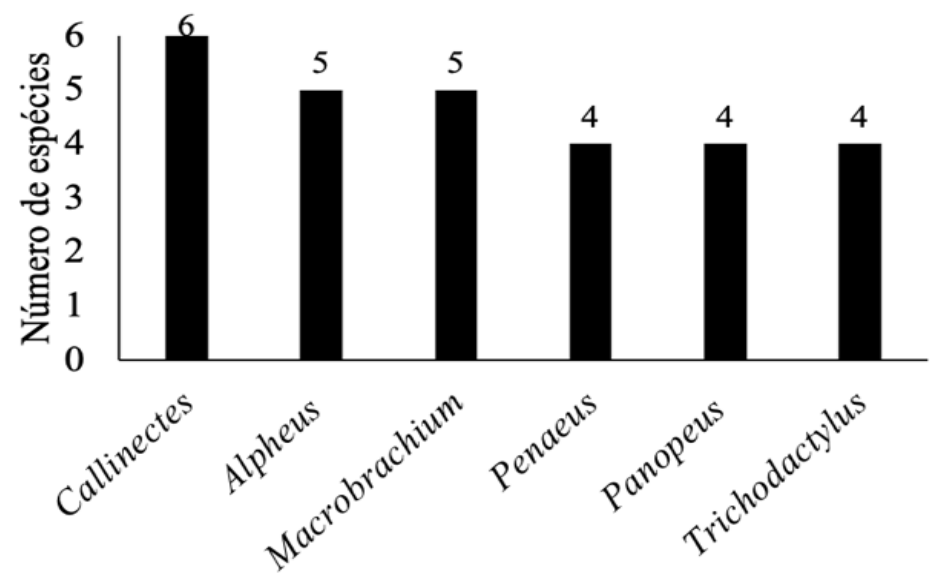

Fonte: Autores.

Considerando a classificação sobre a importância econômica observa-se que $60 \%$ das espécies não possuem valor comercial, $21 \%$ são utilizadas na alimentação humana e/ou para isca na pesca esportiva, $13 \%$ para aquariofilia e $6 \%$ tanto para alimentação humana e/ou para isca na pesca esportiva, como para ornamentação.

\section{3 Área total (Monitoramento + Levantamento bibliográfico)}

Foram registradas 102 espécies pertencentes a 67 gêneros, 31 famílias, sete infraordens e três ordens (Tabela 2).

A ordem que representou o maior número de famílias foi Decapoda (93,5\%), seguida por Isopoda (3,2\%) e Sessilia $(3,2 \%)$ (Figura 11).

Figura 11. Número de famílias por ordens de crustáceos registrados no Complexo Baía-estuário de Santos-São Vicente e canal de Bertioga.

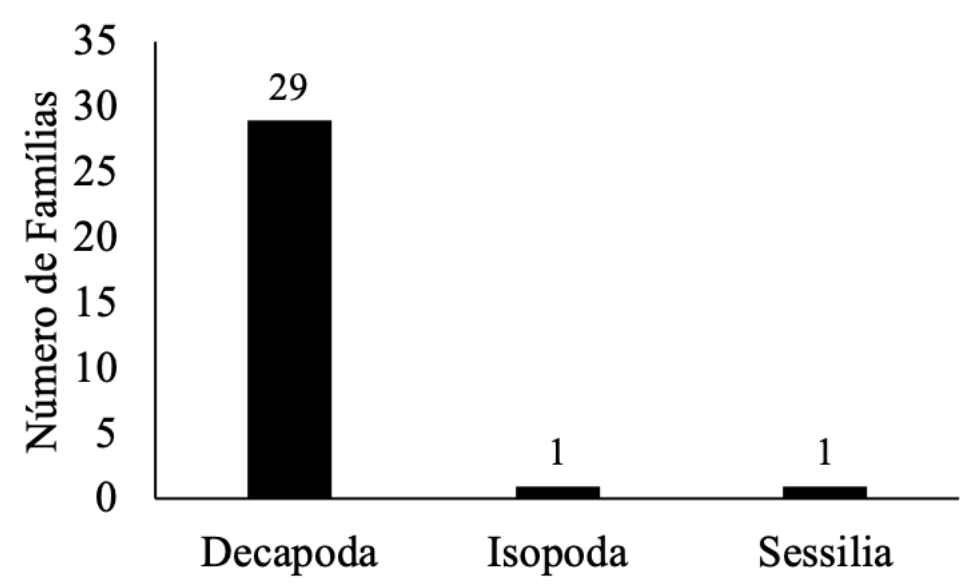

Fonte: Autores.

A infraordem com maior representatividade de famílias foi Brachyura (54,8\%), seguida de Caridea (12,9\%) e Dendrobranchiata (12,9\%), que juntas representaram 80,6\% do total (Figura 12). 
Figura 12. Número de famílias por infraordens de crustáceos registrados no Complexo Baía-estuário de Santos-São Vicente e canal de Bertioga.

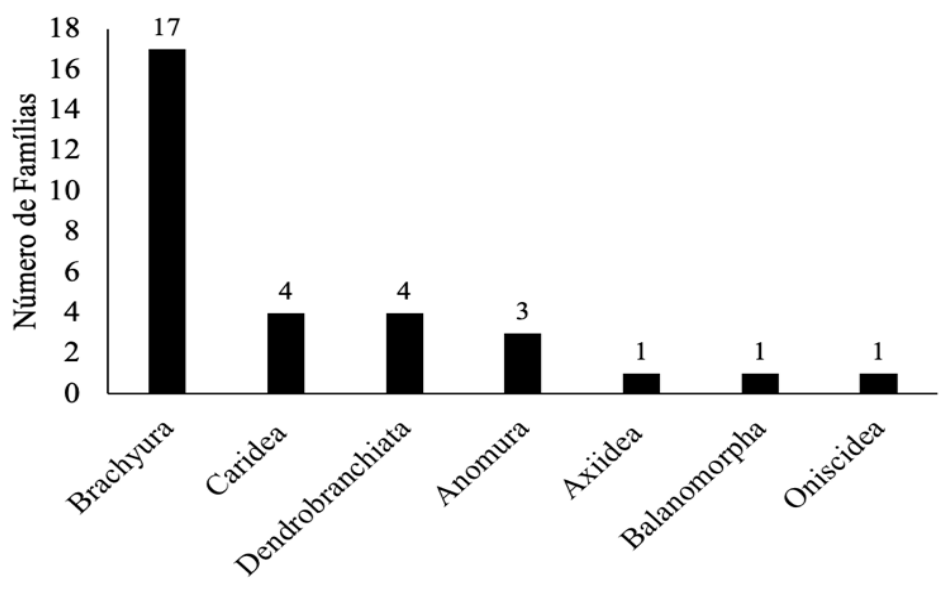

Fonte: Autores.

A infraordem com maior número de espécies foi Brachyura (52\%), seguida de Caridea (22,5\%), que juntas representaram $74,5 \%$ do total de espécies registradas (Figura 13).

Figura 13. Número de espécies por infraordens de crustáceos registrados no Complexo Baía-estuário de Santos-São Vicente e canal de Bertioga.

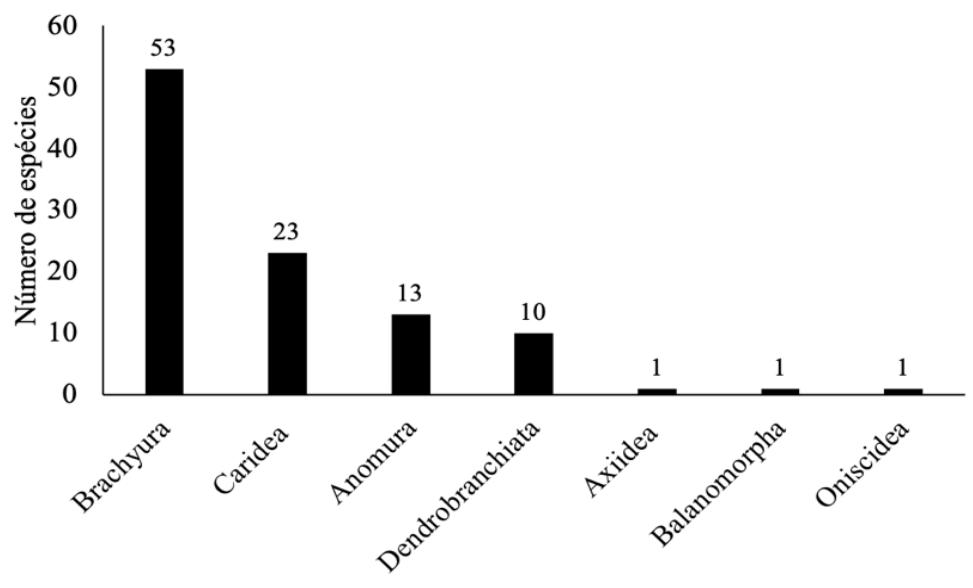

Fonte: Autores.

As famílias que apresentaram o maior número de espécies foram Palaemonida (11,7\%) e Portunidae (11,7\%), seguidas por Panopeidae (8,8\%), Panaeidae (6,9\%) e Alpheidae (6,9\%), que juntas representaram 46\% do total (Figura 14). 
Figura 14. Número de espécies por famílias de crustáceos registrados no Complexo Baía-estuário de Santos-São Vicente e canal de Bertioga.

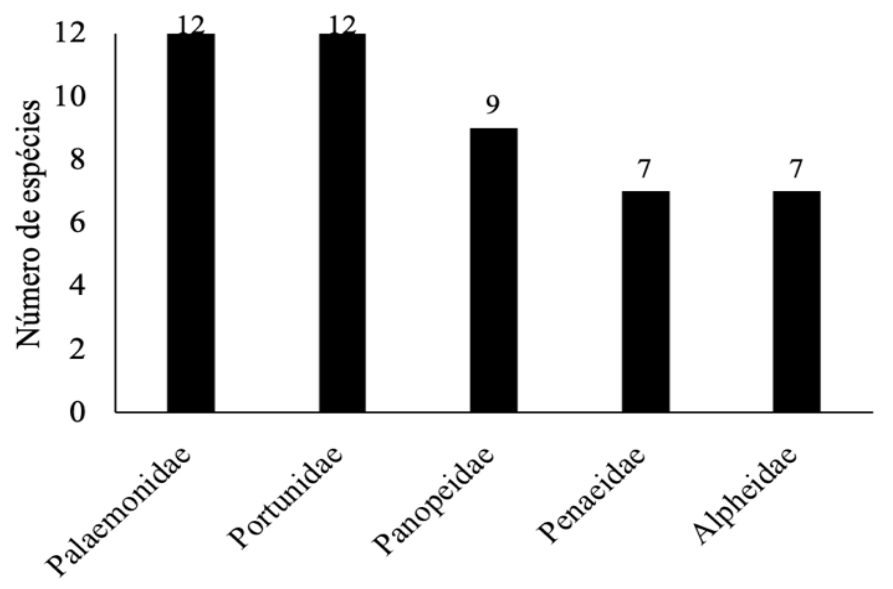

Fonte: Autores.

O gênero com maior representatividade de espécies foi Callinectes (5,9\%), seguido de Alpheus (4,9\%), Macrobrachium (4,9\%), Penaeus (3,9\%), Panopeus (3,9\%) e Trichodactylus (3,9\%), que juntos representaram 27,4\% do total (Figura 15).

Figura 15. Número de espécies por gêneros de crustáceos registrados no Complexo Baía-estuário de Santos-São Vicente e canal de Bertioga.

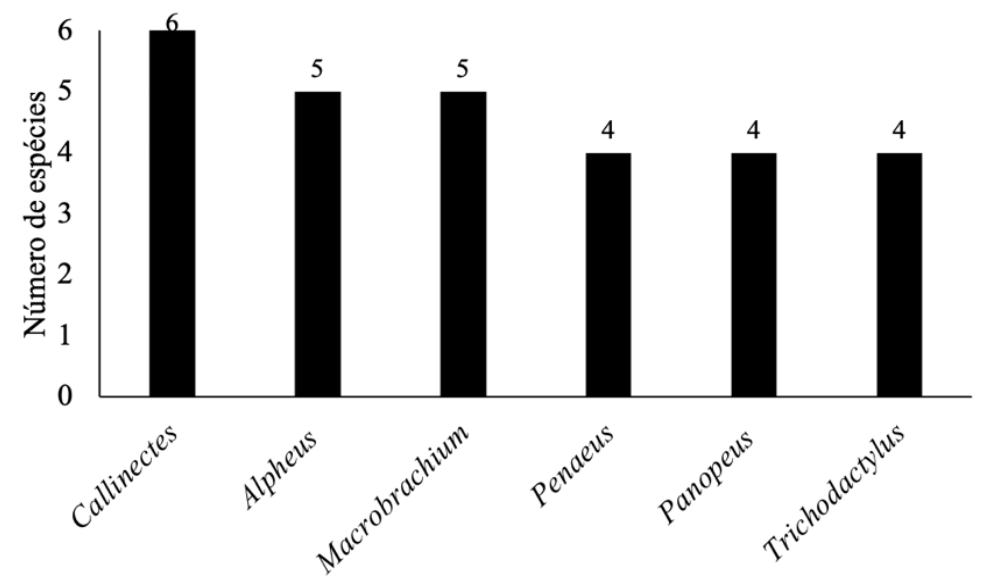

Fonte: Autores.

Com base na classificação sobre a importância econômica observa-se que $61 \%$ das espécies não possuem valor comercial, $21 \%$ são utilizadas na alimentação humana e/ou para isca na pesca esportiva, $14 \%$ para aquariofilia e $6 \%$ tanto para alimentação humana e/ou para isca na pesca esportiva, como para ornamentação.

\section{Discussão}

No monitoramento foram identificadas 18 espécies da ordem Decapoda, caracterizando a Baía de Santos como local de composição taxonômica típica para a região litorânea do sudeste e sul brasileiro. Comparado com outros estudos que utilizaram a mesma metodologia de coleta, porém com diferenças de esforço amostral, verifica-se que a riqueza foi inferior ao relatado por Graça-Lopes et al. (2002) que observaram 28 espécies para a região da Baía de Santos e adjacências (SP), representando baixa 
similaridade na composição (36,11\%). Também foi inferior a Fransozo et al. (2016) que observaram 44 espécies na enseada de Ubatuba (SP), assim como a Costa, Mattos e Machado (2016), que registraram 30 espécies junto a pesca do camarão sete-barbas, na região de Macaé (RJ), e a Severino-Rodrigues, Guerra e Graça-Lopes (2002) que identificaram 41 espécies também associadas a pesca do camarão sete-barbas na Praia do Perequê, Guarujá (SP), representando 33,33\% de similaridade na composição com o presente estudo. Considerando o número total de espécies registradas em estudos acerca da carcinofauna do Complexo Baíaestuário de Santos, São Vicente e Canal de Bertioga observa-se que o monitoramento representou apenas 15,5\% do descrito anteriormente, acrescentando apenas duas espécies (Pagurus longicarpus e Libinia spinosa) a lista consolidada.

A região da Baía de Santos apresenta condições ambientais favoráveis a ocorrência de espécies de crustáceos com ciclo de vida dependente do estuário (Fransozo, Costa, Pinheiro, Santos, \& Mantelatto, 2000, Fransozo et al., 2002, Castilho, Pie, Fransozo, Pinheiro, \& Costa, 2008), sendo esta relação observada na elevada representatividade de Brachyura, seguida por Dendrobranchiata. As infraordens Anomura e Caridea foram as menos expressivas, possivelmente por não possuírem tolerância às condições ambientais da região, como a variação de salinidade e temperatura e por não ocorrerem nas profundidades dos pontos de coleta. Segundo Chávez, Rocha e Vázquez (2019) o siri azul Callinectes sapidus apresenta maior preferência por locais com influência de estuários, como constatado neste estudo e por Severino-Rodrigues (2002) e Reigada et al. (2006) na Baía de Santos e no complexo estuarino Santos-São Vicente respectivamente.

Fransozo et al. (2011) relataram que a elevada abundância de ermitões (Dardanus insignis e Petrochirus diogenes) na região de Ubatuba (SP), favorece a presença de caranguejos porcelanídeos, pois estes habitam o interior das conchas ocupadas por estes ermitões. A ausência dos porcelanídeos no monitoramento indica que a região não apresenta condições ambientais favoráveis para essas infraordens de crustáceos ou os pontos de coleta não coincidiram com as áreas de maior concentração desses grupos. Outro fator que pode contribuir para esta diferenciação, segundo Fransozo et al. (2016), está relacionado com o predomínio de areia muito fina no CBESSCB, o que permite aos ermitões ficarem enterrados e não serem capturados pelo artefato de coleta utilizado. Também é importante ressaltar que os crustáceos possuem preferências por tipos de substrato, porém independentemente do tipo de sedimento ou da concentração de matéria orgânica, as espécies da macrofauna apresentam habilidades de exploração de recursos alimentares em condições ambientais contrastantes, sejam eles recém depositados no substrato ou não. Contudo, as relações de interdependência podem ser influenciadas em diferentes escalas sobre a influência dos fatores ambientais (Quintana, 2008).

É importante considerar que a metodologia de amostragem pode influenciar nos dados de composição da carcinofauna. Neste monitoramento foi adotado o arrasto de fundo como amostrador biológico, porém este artefato restringe a amostragem a espécies associadas ao substrato inconsolidado. Embora eficiente para este grupo de espécies, esta metodologia não captura espécies associadas ao substrato consolidado, mesmo que as espécies sejam abundantes na região, como por exemplo o Guaiá (Menippe nodifrons) que ocorre junto a rochas (Oshiro, 1999), ou o Corrupto (Callichirus major) que habita galerias na região praial (Rodrigues \& Shimizu, 1997), ou ainda a espécie exótica Charybdis hellerii (Sant'Anna et al., 2012) que habita praias arenosas na interface com costões rochosos. Esta relação também pode ser observada através dos resultados de estudos sobre a carcinofauna do CBESSCB, onde por exemplo, verifica-se uma baixa similaridade (0\%) na composição entre Pita et al. (1985) e Micheletti-Flores e Negreiros-Fransozo (1999), onde o primeiro foi realizado utilizando arrasto de fundo e o segundo utilizando espécies associadas a colônias de Phragmatopoma lapidosa (Polychaeta, Sabellariidae). Ou ainda, entre a maior similaridade (36,36\%) entre Reigada et al. (2006) e os resultados deste estudo, ambos realizados com arrasto de fundo.

Fransozo et al. (2016) afirmaram que a pesca realizada com redes não seletivas pode alterar a composição e a diversidade das áreas de atuação das frotas, condições que são típicas da Região da Baía de Santos. Além disso, a pesca é um dos fatores modificadores das comunidades de crustáceos (Kaiser \& Spencer, 1996, Kaiser, Collie, Hall, Jennings, \& Poiner, 2002, Romano et al., 2016). Assim, a pesca na área de estudo deve ser considerada como um fator que pressiona a composição bêntica local por 
ser essencialmente voltada a captura dos camarões. Tal verificação também foi realizada por Severino-Rodrigues et al. (2002) que atribuíram uma importante contribuição da pesca de arrasto na variação espacial e temporal da composição e diversidade da carcinofauna acompanhante da pesca do camarão sete-barbas no litoral de São Paulo.

O camarão sete-barbas X. kroyeri foi a espécie mais abundante, demonstrando a importância da Baía de Santos para este recurso pesqueiro de grande importância econômica regional (Gefe et al., 2004). Entretanto, P. schmitti e P. brasiliensis também foram registradas, porém em menor quantidade. Segundo Simões, Heckler e Costa (2017) no litoral sudeste do Brasil, $X$. kroyeri é a espécie mais abundante no ambiente marinho, enquanto $P$. schmitti, $P$. paulensis e P. brasiliensis são mais representativas no ambiente estuarino. Ressalta-se que a pesca de camarões é intensa na região, mesmo com proteção legal através da portaria IBAMA nº 05 de 1994, a qual proíbe a pesca de arrasto no interior da Baía de Santos.

Além do camarão sete-barbas, nas estações de outono e inverno foram capturados exemplares de $P$. schmitti (camarão branco), sendo sua presença relatada por Santos, Severino-Rodrigues e Vaz-dos-Santos (2008) na Baía de Santos e áreas adjacentes, apresentando pico reprodutivo no período primavera-verão, e recrutamento na área marinha principalmente em fevereiro, coincidindo com o início do defeso camaroeiro. Esta diferenciação pode ser atribuída a metodologia de amostragem de cada estudo, pois em Santos et al. (2008) o desenho amostral apresentou maior amplitude de profundidades quando comparado ao presente estudo, além de terem realizado campanhas mensais de amostragem.

Cabe ressaltar que a pesca do camarão sete-barbas realizada na Baía de Santos pela frota "artesanal” sediada nos municípios de Santos, Guarujá e São Vicente é uma das responsáveis indiretas pela captura do camarão-branco (Graça-Lopes et al., 2002). Assim como a pesca dirigida ao camarão sete-barbas, as capturas direcionadas ao camarão branco ( $P$. schmitti) estão atreladas a uma atividade com elevada captura de fauna acompanhante, incluído a carcinofauna (Santos et al., 2008). Além de capturar de forma eficiente os organismos associados ao fundo, a pesca de arrasto também altera as características físicas do substrato, principalmente na condição de compactação desses fundos (Vianna \& Almeida, 2005, Kaiser et al., 2002, Romano et al., 2016).

A infraordem Brachyura apresentou quatro famílias e oito espécies, sendo as maiores abundâncias numérica e em peso de Callinectes danae e C. ornatus (Portunidae), ficando somente atrás de X. kroyeri (Dendrobranchiata). Em geral, o sudeste do Brasil oferece uma ampla gama de condições que favorecem a reprodução de portunídeos, contribuindo para a abundância relativamente alta destas espécies na área costeira. Porém, variações sazonais na biomassa alteram a representatividade das espécies, sendo esta variação muitas vezes relacionada as migrações reprodutivas e até mesmo pela pesca (Pita et al., 1985, Severino-Rodrigues, Pita, \& Graça-Lopes, 2001, Severino-Rodrigues, Musiello-Fernandes, Moura, Branco, \& Canéo, 2012, Scalco et al., 2018). Embora tenham ocorrido ao longo de todas as estações climáticas com grande representatividade em número e peso, C. danae e C. ornatus foram mais abundantes durante o inverno, assim como X. kroyeri. Diferentemente dos resultados observados em outros estudos sobre a carcinofauna, os dados apresentados corroboraram com Costa et al. (2016), que avaliaram a composição associada a pesca de arrasto em Macaé (RJ), onde X. kroyeri foi a espécie mais abundante. A elevada abundância sazonal de X. kroyeri em profundidades inferiores a 20 metros, geralmente está associada ao incremento do pós-defeso, devido a pressão pesqueira sobre os peneídeos neste o período (Graça-Lopes, Pereira, \& Severino-Rodrigues, 2007, Heckler, Simões, Santos, Fransozo, \& Costa, 2013). Porém é importante destacar que a região da Baía de Santos, assim como as suas áreas adjacentes, contempla movimentos migratórios e reprodutivos que influenciam a maior abundância de $X$. kroyeri no inverno (Severino-Rodrigues et al., 2002).

A abundância de crustáceos decápodes pode sofrer amplas variações espaciais, como observado por Martínez-Guerrero e López-Pérez (2018), que detectaram amplas oscilações tanto na abundância, quanto na riqueza entre regiões no Oceano Pacífico. Devido estas variações é necessário um delineamento amostral criterioso para responder adequadamente as questões sobre levantamentos sistemáticos (De Grave \& Anker, 2017). Considerando o posicionamento geográfico, observa-se que o lado 
de São Vicente apresentou valores de abundância de X. kroyeri superiores ao lado de Santos. Tal condição pode ser atribuída a um conjunto de fatores favoráveis para a espécie, como tipo de fundo e parâmetros ambientais mais estáveis, uma vez que as drenagens dos canais estuarinos apresentam diferenças em relação a suas características. Freire, Bijkerk, Silva, Goya e Carrió (2017) mencionaram que a orientação das barras dos canais estuarinos interfere na deposição do sedimento em ambos os lados da Baía de Santos. O lado de Santos deposita mais sedimento no Leste, enquanto o lado de São Vicente deposita mais sedimento no Sul. Como a barra de Santos apresenta maior volume drenado, consequentemente ocorrerem mais alterações nas variáveis ambientais nesta área.

Neiva, Santos e Jankauskis (1971) e Santos et al. (2008) em levantamento sobre a dinâmica populacional do camarão branco, afirmaram que as proximidades dos canais estuarinos de Santos e São Vicente são áreas de recrutamento devido oferecerem condições de salinidade muito próximas à do estuário interno. Possivelmente a comunidade de crustáceos presentes no lado de Santos sofre maior influência do canal do Porto de Santos, gerando condições mais estressantes para espécies menos tolerantes à poluição, intensa navegação e atividade pesqueira, resultando na preferência dessas espécies pelo lado de São Vicente. Andrade et al. (2015) argumentam que diferenças na granulometria, salinidade e temperatura são responsáveis pela variação de abundância de $C$. danae e $C$. ornatus, o que também pode ser responsável pelas diferenças observadas entre os lados da Baía de Santos. Pita et al. (1985) em estudo no complexo estuarino de Santos, caracterizaram a região da Baía de Santos como importante área para a reprodução de $C$. danae e $C$. ornatus, onde durante o verão, ocupam as regiões rasas da Baía de Santos. Outro aspecto importante a ser considerado, está relacionado ao lado de São Vicente abranger parte da área marinha do Parque Estadual Xixová-Japuí, ficando protegida da pesca e consequentemente favorecendo a presença e abundância de diversas espécies.

O tipo de sedimento e a variação de profundidade são fatores importantes na distribuição sazonal e espacial de crustáceos (Fransozo et al., 2011). Simões, Costa, Fransozo e Castilho (2010) indicaram a variação de profundidade como um dos fatores com maior influência na variação da abundância de X. kroyeri em Ubatuba (SP). O lado de São Vicente e a profundidade média foram as que apresentaram a maior abundância de X. kroyeri, indicando os locais como importante ponto de alimentação, crescimento e maturação da espécie. Da mesma forma, a Baía de Santos pode fornecer condições favoráveis para as atividades tróficas e migratórias de algumas espécies de crustáceos. Segundo Barioto, Stanski, Grabowski, Costa e Castilho (2017) variações sazonais na abundância e no tamanho corporal são características de atividades migratórias, tróficas e reprodutivas entre as regiões estuarinas e marinhas para $P$. schmitti, que possui comportamento migratório e de crescimento semelhantes aos camarões costeiros. A relação com o sedimento também foi relatada por Costa, Fransozo e Negreiros-Fransozo (2005) e Simões et al. (2017), que constataram que a baixa abundância de Sicyonia dorsalis está associada ao tipo de sedimento, predominantemente composto por areia muito fina, limitando a sua presença na região. Ainda segundo estes autores, $S$. dorsalis prefere sedimentos compostos predominantemente de silte e argila e áreas com salinidades altas, diferentemente do observado na Baía de Santos.

Comunidades também podem se estabelecer e agregar em tubos dos sistemas costeiros de tratamento de águas residuais municipais, que descarregam resíduos de águas tratadas no oceano, frequentemente colocados em regiões com sedimentos moles e baixa complexidade de habitat, como é o caso do emissário submarino localizado a oeste da Baía de Santos. A estrutura que o tubo fornece em um habitat de baixa complexidade pode ser atraente para uma ampla variedade de animais, principalmente para os crustáceos, tornando-se efetivamente um habitat de recife artificial que pode se estender por vários quilômetros (Allen, Pecorelli, \& Word, 1976, Love \& York, 2005, Fortune \& Paterson, 2020). Portanto, esses emissários rapidamente se tornam centros de alta produtividade e biodiversidade, que irão sustentar toda a comunidade. Porém, conforme a biomassa ao redor do recife artificial aumenta, os recursos podem se tornar limitados, o que irá forçar alguns indivíduos a expandir a área que habitam ao redor da estrutura ou a abandoná-la em busca de áreas com menos competição (Lowe \& Bray, 2006). Por outro lado, o 
emissário também poderá atuar como um impedimento para algumas espécies que normalmente usam habitats de baixo relevo, devido à maior abundância de predadores que podem residir nesse local. Assim, o comportamento dos indivíduos, tanto intra quanto interespecífico, ao redor do recife artificial podem ser complexos.

Vários estudos condicionaram a presença de ilhas, baías, enseadas e estuários como locais que favorecem a dinâmica de populações de decápodes (Negreiros-Fransozo, Reigada, \& Fransozo, 1992, Negreiros-Fransozo, Fransozo, Mantelatto, \& Santos, 1997, Negreiros-Fransozo et al., 1998, Negreiros-Fransozo, Nakagaki, \& Reigada, 1999, Braga, Fransozo, Bertini, \& Fumis, 2005). O lado de Santos se diferencia pela atividade de dragagem do canal de navegação do Porto de Santos, que apesar de gerar uma condição estressante para a comunidade aquática, pode ser responsável em gerar condições que favorecem organismos mais tolerantes a essa interferência. Reigada et al. (2006) em estudo realizado com siris na Baía de Santos, observaram uma elevada concentração desses crustáceos em diversos locais da baía, inclusive do lado de Santos. Os autores atribuíram essa condição a fatores como temperatura, salinidade e profundidade, que apresentaram maiores amplitudes em determinados locais da Baía de Santos. Neste sentido, Silva, Sancinetti, Fransozo, Azevedo e Costa (2014) e Andriguetto-Filho, Natividade, Brandini e de Teixeira (2016) destacaram as variações na temperatura do fundo e transparência da água como os principais fatores que controlaram a distribuição e o ciclo de vida dos camarões marinhos, assim como de outros crustáceos, também sujeitos as variações de salinidade. Tal fato pode estar associado às condições oceanográficas atuantes na Baía de Santos, que controlam as variações sazonais na biomassa e podem estar relacionadas com as migrações costeiras de crustáceos sob influência de correntes (Andriguetto-Filho et al., 2016), sendo a hidrografia local responsável por alterar os padrões de ciclo de vida e distribuição das espécies.

\section{Considerações Finais}

Apesar da elevada antropização do Complexo Baía-estuário de Santos-São Vicente e Canal de Bertioga (CBESSCB) (Lamparelli et al., 2001, Medeiros \& Bícego, 2004, CETESB, 2007, Martins et al., 2008, Siqueira et al., 2012), este é caracterizado como de extrema importância biológica (MMA, 2018), apresentando elevada riqueza de crustáceos. Mesmo com a facilidade de acesso e sua relevância geoeconômica, o CBESSCB carece de estudos sobre a composição taxonômica, assim como aspectos da estrutura de comunidade e populações de crustáceos.

Neste sentido, os resultados apresentados sumarizaram a composição do CBESSCB, respondendo inicialmente as ações prioritárias sugeridas pelo Ministério do Meio Ambiente (MMA, 2018). Porém é importante destacar que são necessários novos levantamentos, utilizando diferentes métodos de captura em toda a área do complexo, contemplando também os ciclos sazonais para a obtenção de registros adequados da carcinofauna local. De forma incipiente, os resultados sobre a abundância dos crustáceos da Baía de Santos indicaram a importância socioeconômica da região para a pesca de crustáceos, sendo necessário estudos complementares para a adoção de políticas de conservação que considerem as atividades portuárias e pesqueiras desenvolvidas.

\section{Referências}

Abessa, D. M. S. (2002). Avaliação da qualidade de sedimentos do Sistema Estuarino de Santos, SP, Brasil. [tese de doutorado, Instituto Oceanográfico da Universidade de São Paulo, Brasil. https://repositorio.usp.br/item/001311154

Abessa, D. M. S., Carr, R. S., Sousa, E. C. P. M., Rachid, B. R. F., Zaroni, L. P., Gasparro, M. R., Pinto, Y. A., Bícego, M. C., Hortellani, M. A., Sarkis, L. E. S., \& Muniz, P. (2008). Integrative Ecotoxicological Assessment of a Complex Tropical Estuarine System. Em: TN, Hoffer (Ed.), Marine Pollution: New Research (cap. 4, pp. 1-36). Nova Science Publishers.

Allen, M. J., Pecorelli, H., \& Word, J. (1976). Marine organisms around outfall pipes in Santa Monica Bay. Journal Water Pollution Control Federation, 18811893. https://www.jstor.org/stable/25039959 
Almeida, A. O., \& Coelho, P. A. (2008). Estuarine and marine brachyuran crabs (Crustacea: Decapoda) from Bahia, Brazil: checklist and zoogeographical considerations. Latin American Journal of Aquatic Research, 36(2), 183-222. https://doi.org/10.3856/vol36-issue 2-fulltext-4

Almeida, A. O., Costa-Souza, A. C., Cunha, A. M., Santos, P. S., Oliveira, M. V., \& Soledade, G. O. (2013). Estuarine caridean shrimps (Crustacea: Decapoda) from Ilhéus, Bahia, Brazil: Updated checklist and a key for their identification. Check List, 9(6), 1396-1405. https://doi.org/10.15560/9.6.1396

Almeida, A. O., Guerrazzi, M. C., \& Coelho, P. A. (2007). Stomatopod and decapod crustaceans from Camamu Bay, state of Bahia, Brazil. Zootaxa, 1553(1), 1-45. https://doi.org/10.11646/zootaxa.1553.1.1

Almeida, A. O., Souza, G. B. G., Boehs, G., \& Bezerra, L. E. A. (2010). Shallow-water anomuran and brachyuran crabs (Crustacea: Decapoda) from southern Bahia, Brazil. Latin American Journal of Aquatic Research, 38(3), 329-376. https://doi.org/10.3856/vol38-issue3-fulltext-2

Almeida, A. O., Terossi, M., Buranelli, R. C., Castilho, A. L., Costa, R. C., Zara, F. J., \& Mantelatto, F. L. (2018). Checklist of decapods (Crustacea) from the coast of São Paulo State (Brazil) supported by integrative molecular and morphological data: II. Infraorder Caridea: family Alpheidae. Zootaxa, 4450(3), 331358. https://doi.org/10.11646/zootaxa.4450.3.2

Alves, D. F. R., Barros-Alves, S. P., Cobo, V. J., Lima, D. J. M., \& Fransozo, A. (2012). Checklist of the brachyuran crabs (Crustacea: Decapoda) in the rocky subtidal of Vitória Archipelago, southeast coast of Brazil. Check List, 8(5), 940-950. https://doi.org/10.15560/8.5.940

Alves, D. F. R., Cobo, V. J., \& Melo, G. A. S. (2006). Extension of the geographical distribution of some brachyuran and porcellanid decapods (Crustacea) to the coast of the State of São Paulo, Brazil. Revista Brasileira de Zoologia, 23(4), 1280-1283. http://doi.org/10.1590/S0101-81752006000400045

Alves, M. L., Ramos-Porto, M., \& Viana, G. F. S. (2008). Checklist of the decapods (Crustacea) from the Fernando de Noronha Archipelago, Brazil. Zootaxa, 1881(1), 43-68. https://doi.org/10.11646/zootaxa.1881.1.2

Andrade, L. S., Antunes, M., Lima, P. A., Furlan, M., Frameschi, I. F., \& Fransozo, A. (2015). Reproductive features of the swimming crab Callinectes danae (Crustacea, Portunoidea) on the subtropical coast of Brazil: a sampling outside the estuary. Brazilian Journal of Biology, 75(3), 692702.https://doi.org/10.1590/1519-6984.21513

Andriguetto-Filho, J. M., Natividade, C. D., Brandini, F. P., \& de Teixeira, R. A. (2016). Local hydrography and fishing drive life cycle strategies and population dynamics of the sea-bob shrimp Xiphopenaeus kroyeri in a coastal subtropical environment in Brazil. Hydrobiologia, 771(1), 207-225. https://doi.org/10.1007/s10750-015-2631-4

Anker, A., Tavares, M., \& Mendonça, J. B. (2016). Alpheid shrimps (Decapoda: Caridea) of the Trindade \& Martin Vaz Archipelago, off Brazil, with new records, description of a new species of Synalpheus and remarks on zoogeographical patterns in the oceanic islands of the tropical southern Atlantic. Zootaxa, 4138(1), 1-58. https://doi.org/10.11646/zootaxa.4138.1.1

Barioto, J. G., Stanski, G., Grabowski, R. C., Costa, R. C., \& Castilho, A. L. (2017). Ecological distribution of Penaeus schmitti (Dendrobranchiata: Penaeidae) juveniles and adults on the southern coast of São Paulo state, Brazil. Marine Biology Research, 13(6), 693-703. https://doi.org/10.1080/17451000.2017.1287923

Bertini, G., Fransozo, A., \& Melo, G. A. S. (2004). Biodiversity of brachyuran crabs (Crustacea: Decapoda) from non-consolidated sublittoral bottom on the northern coast of São Paulo State, Brazil. Biodiversity \& Conservation, 13(12), 2185-2207. https://doi.org/ 10.11646/zootaxa.1956.1.1

Boos, H., Buckup, G. B., Buckup, L., Araujo, P. B., Magalhães, C., Almerão, M. P., Santos, R. A., \& Mantelatto, F. L. (2012). Checklist of the Crustacea from the state of Santa Catarina, Brazil. Check List, 8(6), 1020-1046. https://doi.org/10.15560/8.6.1020

Braga, A. A., Fransozo, A., Bertini, G., \& Fumis, P. B. (2005). Composição e abundância dos caranguejos (Decapoda, Brachyura) nas regiões de Ubatuba e Caraguatatuba, litoral norte paulista, Brasil. Biota Neotropica, 5(2), 45-78. http://doi.org/10.1590/S1676-06032005000300004.

Buruaem, L. M., Hortellani, M. A., Sarkis, J. E., Costa-Lotufo, L. V., \& Abessa, D. M. (2012). Contamination of port zone sediments by metals from Large Marine Ecosystems of Brazil. Marine Pollution Bulletin, 64(3), 479-488. http://doi.org/10.1016/j.marpolbul.2012.01.017. PMid:22306311.

Carvalho-Neta, R. N. F., Oliveira-Mota, T. D. S., Oliveira, S. R. S., Torres Junior, A. R., Silva Cardoso, W., Santos, D. M. S., Batista, W. S., Serra, I. M. R. S., \& Brito, N. M. (2019). Biochemical and morphological responses in Ucides cordatus (Crustacea, Decapoda) as indicators of contamination status in mangroves and port areas from northern Brazil. Environmental Science and Pollution Research, 26(16), 15884-15893. https://doi.org/10.1007/s11356-019-04849-0

Castilho, A. L., Pie, M. R., Fransozo, A., Pinheiro, A. P., \& Costa, R. C. (2008). The relationship between environmental variation and species abundance in shrimp community (Crustacea: Decapoda: Penaeoidea) in south-eastern Brazil. Journal of the Marine Biological Association of the United Kingdom, 88, 119. 123. https://doi.org/10.1017/S0025315408000313

Castro, B. M., Miranda, L. B., Silva, L. S., Fontes, R. F. C., Pereira, A. F. \& Coelho, A. L. (2008). Processos Físicos: Hidrografia, Circulação e Transporte. Em: MAS, Pires-Vanin (Ed.), Oceanografia de um ecossistema subtropical - São Paulo: Edusp. https://repositorio.usp.br/item/001728923

Chávez, R., Rocha, A., \& Vázquez, H. (2019). Aspectos ecológicos de los estadios juveniles de Callinectes sapidus (Crustacea: Portunidae) en un estuario ciego del Golfo de México. Caldasia, 41(2), 422-432. https://doi.org/10.15446/caldasia.v41n2.70500

Cesar, A., Pereira, C. D. S., Santos, A. R., Abessa, D. M. S., Fernández, N., Choueri, R. B., \& Delvalls, T. A. (2006). Ecotoxicological assessment of sediments from the Santos and São Vicente Estuarine System Brazil. Brazilian Journal of Oceanography, 54(1), 55-63. http://doi.org/10.1590/S1679-87592006000100005

CETESB - Companhia Ambiental do estado de São Paulo. (2007). Poluição das águas no estuário e Baía de Santos. Relatório técnico, 71p.

Choueri, R. B. (2008). Armonización de protocolos para la evaluación de sedimentos y materiales dragados en zonas costeras del Atlántico. [tese de Doutorado, Universidade de Cádiz, Cádiz, Espanha]. https://rodin.uca.es/handle/10498/15692

Coelho, P. A., Almeida, A. O., \& Bezerra, L. E. A. (2008). Checklist of the marine and estuarine Brachyura (Crustacea: Decapoda) of northern and northeastern Brazil. Zootaxa, 1956(1), 1-58. https://doi.org/10.11646/zootaxa.1956.1.1 
Coelho, P. A., Almeida, A. O., Bezerra, L. E. A., \& Souza-Filho, J. F. (2007). An updated checklist of decapod crustaceans (infraorders Astacidea, Thalassinidea, Polychelida, Palinura, and Anomura) from the northern and northeastern Brazilian coast. Zootaxa, 1519(1), 1-16. https://doi.org/10.11646/zootaxa.1519.1.1

Coelho, P. A., Almeida, A. O., Souza-Filho, J. F., Bezerra, L. E. A., \& Giraldes, B. W. (2006). Diversity and distribution of the marine and estuarine shrimps (Dendrobranchiata, Stenopodidea and Caridea) from North and Northeast Brazil. Zootaxa, 1221(1): 41-62. https://doi.org/10.11646/zootaxa.1221.1.5

Cortez, F. S., Silva Souza, L., Guimarães, L. L., Almeida, J. E., Pusceddu, F. H., Maranho, L. A., Mota, L. G., Nobre, C. R., Moreno, B. B., Abessa, D. M. S., Cesar, A., Santos, A. R., \& Seabra Pereira, C. D. (2018). Ecotoxicological effects of losartan on the brown mussel Perna perna and its occurrence in seawater from Santos Bay (Brazil). Science of the Total Environment, 637: 1363-1371. https://doi.org/10.1016/j.scitotenv.2018.05.069

Costa, J. A., Deus, S. R. F., \& Rodrigues, E. S. (2020). Caracterização de Brachyura e Porcellanidae da Ilha Das Palmas, Santos-SP, com base em imagens digitais. Unisanta BioScience, 9(2), 96-113. https://periodicos.unisanta.br/index.php/bio/article/view/2233/0

Costa, R. C., Fransozo, A., \& Negreiros-Fransozo, M. L. (2005). Ecology of the rock shrimp Sicyonia dorsalis Kingsley, 1878 (Crustacea: Sicyoniidae) in a subtropical region of Brazil. Gulf and Caribbean Research, 17, 49-56. https://doi.org/10.18785/gcr.1701.05

Costa, R. C., Fransozo, A., Melo, G. A. S., \& Freire, F. A. M. (2003). Chave ilustrada para identificação dos camarões dendrobranchiata do litoral norte do estado de São Paulo, Brasil. Biota Neotropica, 3(1), 1-12. https://doi.org/10.1590/S1676-06032003000100011

Costa, T. V., Mattos, L. A., \& Machado, N. J. B. (2016). Estrutura populacional de Macrobrachium amazonicum em dois Lagos de Várzea da Amazônia, Boletim Instituto de Pesca, 42(2), 281-293. https://doi.org/10.20950/1678-2305.2016v42n2p281

D'Incao, F. (1995a). Brazilian rock shrimps of the genus Sicyonia (Decapoda: Sicyoniidae). Nauplius, 3, 101-125. http://www.crustacea.org.br/wpcontent/uploads/2014/02/nauplius-v03n1a09.DIncao.pdf

D'incao, F. (1995b). Taxonomia e padrões distribucionais e ecológicos dos Dendrobranchiata (Crustacea: Decapoda) do litoral brasileiro. [tese de Doutorado, Universidade Federal do Paraná, Curitiba, Brazil]. http://livros01.livrosgratis.com.br/cp110530.pdf

De Grave, S., \& Anker, A. (2017). An annotated checklist of marine caridean and Stenopodidean shrimps (Malacostraca: Decapoda) of the Caribbean coast of Panama. Nauplius, 25, e2017015. https://doi.org/10.1590/2358-2936e2017015

Decreto do Estado de São Paulo (2008). No. 53,526 de Outubro 08, 2008. Governo do Estado de São Paulo. http://www.icmbio.gov.br/cepsul/images/stories/. legislacao/Decretos/2008/dec_53526_2008_criaapamarinhalitoralcentro_sp.pdf.

Ferreira, R. S., Vieira, R. R. R., \& D'Incao, F. (2010). The marine and estuarine shrimps of the Palaemoninae (Crustacea: Decapoda: Caridea) from Brazil. Zootaxa, 2606: 1-24. https://doi.org/10.11646/zootaxa.2606.1.1

Fortune, I. S., \& Paterson, D. M. (2020). Ecological best practice in decommissioning: a review of scientific research. ICES Journal of Marine Science, 77(3), 1079-1091. https://doi.org/10.1093/icesjms/fsy130

Fransozo A., Costa R. C., Mantelatto F. L. M., Pinheiro M. A. A., \& Santos, S. (2002). Composition and abundance shrimp species (Penaeidea and Caridea) in Fortaleza bay, Ubatuba, São Paulo, Brasil. Em: EE. Briones \& F. Alvarez (Eds.), Modern approaches to the study of Crustacea. México: Kluwer Academic Press (pp.117-124). https://doi.org/10.1007/978-1-4615-0761-1_19

Fransozo, A. Sousa, A. N., Rodrigues, G. F., Telles, J. N., Fransozo, V., \& Fransozo, M. L. N. (2016). Crustáceos decápodes capturados na pesca do camarãosete barbas no sublitoral não consolidado do litoral norte do estado de São Paulo, Brasil. Boletim do Instituto de Pesca, 42(2), 369-386. https://doi.org/10.20950/1678-2305.2016v42n2p369

Fransozo, A., Costa, R. C., Pinheiro, M. A. A., Santos, S., \& Mantelatto, F. L. M. (2000). Juvenil recruitment of the seabob Xiphopenaeus kroyeri (Heller, 1862) (Decapoda, Penaeidae) in the Fortaleza Bay, Ubatuba, SP, Brazil. Nauplius, 8(2), 179-184. http://crustacea.org.br/wp-content/uploads/2014/02/naupliusv08n2a02.Fransozo.et_.al_.pdf

Fransozo, A., Fernandes-Góes, L. C., Fransozo, V., Góes, J. M., Cobo, V. J., Teixeira, G. M., \& Gregati, R. A. (2011). Marine Anomurans (Decapoda) from the Non-Consolidated Sublittoral Bottom at the Southeastern Coast of Brazil. Crustaceana, 84(4), 435-450. https://doi.org/10.1163/001121611X559251

Freire, I. B., Bijkerk, R., Silva, C. M., Goya, S. C., \& Carrió, J. A. (2017). Incremento da erosão nas praias da Baía de Santos. Revista Mundo Investigación, 2 (2), 75. file:///Users/amandacarminatto/Downloads/Incrementodaerosaonaspraiasdabaiadesantos.pdf

Gefe, W. E., Amorim, L. F. C., Amorim, A. C., \& Amorim, F. A. (2004) Aspectos sócio-econômicos da pesca artesanal na Região da Baixada Santista. IV Congresso Brasileiro de Pesquisas Ambientais e Saúde, 2004, 13-21, S.P., Anais... Núcleo de Pesquisas Ambientais da Baixada Santista. 1CD-ROM.

Graça-Lopes, R., Pereira, E., \& Severino-Rodrigues, E. (2007). Aportes ao Conhecimento da Biologia e da Pesca do Camarão-sete-barbas (Xiphopenaeus kroyeri Heller, 1862) no Litoral do Estado de São Paulo, Brasil. Boletim Instituto de Pesca, 33(1), 63-84. https://209.124.77.230/index.php/bip/article/view/741

Graça-Lopes, R., Puzzi, A., Severino-Rodrigues, E., Bartolotto, A. S., Guerra, D. S. F., \& Figueiredo, K. T. B. (2002). Comparação entre a produção de camarão sete-barbas e de fauna acompanhante pela frota de pequeno porte sediada na Praia de Perequê, Estado de São Paulo, Brasil. Boletim Instituto de Pesca, 28(2), 189-194. https://www.pesca.sp.gov.br/boletim/index.php/bip/article/view/28_2_189-194

Guterres, L. F. R. (2003). Sistemática e biogeografia do gênero Alpheus fabricius, 1798, do Atlântico Ocidental (Decapoda, Caridea, Alpheidae). [tese de doutorado, Universidade de São Paulo, São Paulo, Brazil].

Heckler, G., Simões, S., Santos, A., Fransozo, A., \& Costa, R. (2013). Population dynamics of the seabob shrimp Xiphopenaeus kroyeri (Dendrobranchiata, Penaeidae) in south-eastern Brazil. African Journal of Marine Science, 35(1), 17-24. https://doi.org/10.2989/1814232X.2013.769901

Holthuis, L. B. (1993). The recent genera of the Caridean and Steno-podidean shrimps (Crustacea, Decapoda), with an appendix on the Order Amphionidacea. Leiden: National Natural History Museum. https://doi.org/10.5962/bhl.title.152891 
IBAMA - Instituto Brasileiro do Meio Ambiente e dos Recursos Naturais Renováveis. (1994). Portaria Ibama $\mathrm{n}^{\circ} 5$, 27 de janeiro de 1994. https://www.icmbio.gov.br/cepsul/images/stories/legislacao/Portaria/1994/p_ibama_05_n_1994_cercoflutuante.pdf.

Kaiser, M. J., Collie, J. S., Hall, S. J., Jennings, S., \& Poiner, I. R. (2002). Modification of marine habitats by trawling activities: prognosis and solutions. Fish and Fisheries, 3(2), 114-136. https://doi.org/10.1046/j.1467-2979.2002.00079.x

Kaiser, M. J., \& Spencer, B. E. (1996). The effects of beam-trawl disturbance on infaunal communities in different habitats. Journal of Animal Ecology, 65, 348358. https://www.jstor.org/stable/5881

Kim, B. S. M., Salaroli, A. B., Lima Ferreira, P. A., Sartoretto, J. R., de Mahiques, M. M., \& Figueira, R. C. L. (2016). Spatial distribution and enrichment assessment of heavy metals in surface sediments from Baixada Santista, Southeastern Brazil. Marine Pollution Bulletin, 103(1-2), 333-338. https://doi.org/10.1016/j.marpolbul.2015.12.041

Lamparelli, M. L., Costa, M. P., Prósperi, V. A., Bevilácqua, J. E., Araújo, R. P. A., Eysink, G. G. L., \& Pompéia, S. (2001). Sistema Estuarino de Santos e São Vicente. Relatório Técnico CETESB., 178p.

Lima, F. A., \& Martinelli-Lemos, J. M. (2019). Checklist of the Brachyura of the Brazilian Amazon Coastal Zone and knowledge status of their larval development. Zootaxa, 4646(2), 301-321. https://doi.org/10.11646/zootaxa.4646.2.6

Love, M. S., \& York, A. (2005). A comparison of the fish assemblages associated with an oil/gas pipeline and adjacent seafloor in the Santa Barbara Channel, Southern California Bight. Bulletin of $\quad$ Marine $\quad$ Science, https://www.ingentaconnect.com/content/umrsmas/bullmar/2005/00000077/00000001/art00007

Lowe, C. G., \& Bray, R. N. (2006). Fish movement and activity patterns. In The Ecology of California Marine Fishes. University of California Press, Berkeley, CA.

Luederwaldt, H. (1919). Os manguezais de Santos. Revista Museu Paulista, 11, 309-408.

Mandaji, D. D. S. (2008). Emissário submarino de Santos: contribuição nos sedimentos de fundo para Al, $\mathrm{Mg}, \mathrm{K}, \mathrm{Ca}, \mathrm{Fe}, \mathrm{Ti}, \mathrm{Na}, \mathrm{Si}, \mathrm{Ba}, \mathrm{Cu}, \mathrm{Zn}$, $\mathrm{Cr}, \mathrm{Mn}$, Co, $\mathrm{Ni}$ $e$ S. [dissertação de mestrado, Universidade de São Paulo, São Paulo, Brasil. https://www.teses.usp.br/teses/disponiveis/44/44142/tde-02092008-163850/en.php

Mantelatto, F. L., Faria, F. C. R., Biagi, R., \& Melo, G. A. S. (2004). Majoid crabs community (Crustacea: Decapoda) from infralittoral rocky/sandy bottom of Anchieta Island, Ubatuba. Brazilian Archives of Biology and Technology, 47(2), 273-279. http://doi.org/10.1590/S1516-89132004000200015

Mantelatto, F. L., Tamburus, A. F., Magalhães, T., Buranelli, R. C., Terossi, M., Negri, M., Castilho, A. L., Costa, R. C., \& Zara, F. J. (2020). Checklist of decapod crustaceans from the coast of the São Paulo state (Brazil) supported by integrative molecular and morphological data: III. Infraorder Brachyura Latreille, 1802. Zootaxa, 4872(1), 1-108. https://doi.org/10.11646/zootaxa.4872.1.1

Mantelatto, F. L., Terossi, M., Negri, M., Buranelli, R. C., Robles, R., Magalhães, T., Tamburus, A. F., Rossi, N., \& Miyazaki, M. J. (2017). DNA sequence database as a tool to identify decapod crustaceans on the São Paulo coastline. Mitochondrial DNA Part A, $29(5)$, 805-815. https://doi.org/10.1080/24701394.2017.1365848

Martínez-Guerrero, B., \& López-Pérez, A. (2018). Richness and large-scale distribution of marine benthic caridean shrimps (Decapoda: Caridea) from the Eastern Tropical Pacific. Nauplius, 26, e2018035. https://doi.org/10.1590/2358-2936e2018035

Martins, C. D. C., Gomes, F. B. A., Ferreira, J. A., \& Montone, R. C. (2008). Organic markers of sewage contamination in surface sediments from Santos Bay, São Paulo. Química Nova, 31(5),1008-1014. https://doi.org/10.1590/S0100-40422008000500012

Medeiros, P. M., \& Bicego, M. C. (2004). Investigation of natural and anthropogenic hydrocarbon inputs in sediments using geochemical markers. I. Santos, SP-Brazil. Marine Pollution Bulletin, 49(9-10), 761-769. https://doi.org/10.1016/j.marpolbul.2004.06.001

Melo, G. A. S. (1996). Manual de identificação dos Brachyura (Caranguejos e Siris) do litoral Brasileiro. Plêiade/FAPESP. https://repositorio.usp.br/single.php?_id=000921427

Melo, G. A. S. (1999). Manual de identificação dos Crustacea Decapoda do litoral Brasileiro: Anomura, Thalassinidea e Astacidea. Plêiade/FAPESP. https://decapoda.nhm.org/pdfs/29504/29504-001.pdf

Micheletti-Flores, C. V., \& Negreiros-Fransozo, M. L. (1999). Porcellanid crabs (Crustacea, Decapoda) inhabiting sand reefs built by Phragmatopoma lapidosa (Polychaeta Sabellariidae) at Paranapuã beach, São Vicente, SP, Brazil. Revista Brasileira de Biologia, 59(1), 63-73. https://doi.org/10.1590/S003471081999000100009

Ministério do Meio Ambiente. (2018). Áreas Prioritárias para a Conservação, Uso Sustentável e repartição de Benefícios da Biodiversidade Brasileira: 2a Atualização - Decreto no 5092 de 21 de maio de 2004. Ministério do Meio Ambiente. Secretaria de Biodiversidade e Florestas, Brasília. http://areasprioritarias.mma.gov.br

Moreira, P. S., Paiva Filho, A. M., Okida, C. M., Schmiegelow, J. M. M., \& Giannini, R. (1988). Bioecologia de crustáceos decápodos, braquiúros, no sistema baía-estuário de Santos e São Vicente, SP: 1. Ocorrência e composição. Boletim do instituto Oceanográfico, 36(1-2), 55-62. http://doi.org/10.1590/S037355241988000100006

Mossolin, E. C., Pileggi, L. G., \& Mantelatto, F. L. (2016). Crustacea, Decapoda, Palaemonidae, Macrobrachium Bate, 1868, São Sebastião Island, state of São Paulo, southeastern Brazil. Check List, 6(4), 605-613. https://doi.org/10.15560/6.4.605

Negreiros-Fransozo, M. L., \& Nakagaki, J. M. (1998). Differential benthic occupation by crabs in the Ubatuba Bay, São Paulo, Brazil. Journal of Shellfish Research, 293-297. https://repositorio.unesp.br/handle/11449/19184

Negreiros-Fransozo, M. L., Fransozo, A., Mantelatto, F. L. M., \& Santos, S. (1997). Anomura species (Crustacea, Decapoda) and their ecological distribution at Fortaleza bay sublitoral, Ubatuba, São Paulo, Brazil. Iheringia Serie Zoológica, 83, 187-194. http://biostor.org/reference/80140 
Negreiros-Fransozo, M. L., Nakagaki, J. M., \& Reigada, A. L. D. (1999). Seasonal occurrence of decapods in shallow waters of a subtropical area. Em: Klein JCVV \& FR Schram (Eds.) The Biodiversity Crisis and Crustacea, Crustacean Issues (vol. 12, pp. 351-361).

Negreiros-Fransozo, M. L., Reigada, A. L. D., \& Fransozo, A. (1992). Braquiúros (Crustacea, Decapoda) dos sedimentos sublitorais da Praia da Enseada, Ubatuba (SP). Boletim Instituto de Pesca, 19 (único), 17-22. https://www.pesca.agricultura.sp.gov.br/boletim/index.php/bip/article/view/19_01_unico_17-22

Neiva, G. S., Santos, E. P., \& Jankauskis, V. (1971). Análise preliminar da população de camarão-legítimo Penaeus schmitti, Burkenroad, 1936, na Baía de Santos - Brasil. Boletim do Instituto de Pesca, 1(2), 7-14. https://www.pesca.sp.gov.br/sumario1.htm

Nunes, L. H., Greco, R., \& Marengo, J. A. (2019). Climate Change in Santos Brazil: Projections, Impacts and Adaptation Options. Springer.

Oshiro, L. M. Y. (1999). Aspectos reprodutivos do caranguejo guaiá Menippe nodifrons, Stimpson, 1859 (Crustácea, Decapoda, Xanthidae) da Baía de Sepetiba (RJ). Revista Brasileira de Zoologia, 16(3), 827-834. http://doi.org/10.1590/S0101-81751999000300019.

Pachelle, P. P. G., Anker, A., Mendes, C. B., \& Bezerra, L. E. A. (2016). Decapod crustaceans from the state of Ceará, northeastern Brazil: an updated checklist of marine and estuarine species, with 23 new records. Zootaxa, 4131(1), 1-63. https://doi.org/10.11646/zootaxa.4131.1.1

Pérez-Farfante, I., \& Kensley, B. (1997). Penaeoid and sergestoid shrimps and prawns of the world. Keys and diagnoses for the families and genera. Paris: Éditions du Muséum National d'Histoire Naturelle. https://www.journals.uchicago.edu/doi/abs/10.1086/393020

Pita, J. B., Rodrigues, E. S., Graça Lopes, R., \& Coelho, J. A. P. (1985). Levantamento da família Portunidade (Crustácea, Decapoda, Brachyura) no complexo baia-estuário de Santos, São Paulo, Brasil. Boletim do Instituto de Pesca, 12(3), 153-162. https://www.pesca.agricultura.sp.gov.br/boletim/index.php/bip/article/view/sumario_12_153-162

Quintana, C. O. (2008). Relações entre as comunidades bênticas e a matéria orgânica sedimentar: respostas à qualidade dos recursos alimentares e influência na diagênese recente. (tese de doutorado, Instituto Oceanográfico da Universidade de São Paulo, São Paulo, Brasil). https://www.teses.usp.br/teses/disponiveis/21/21131/tde-18062009-162309/en.php

Reigada, A. L. D., Sant'anna, B. S., Zangrande, C. M., \& Costa, R. C. (2006). Macrocrustaceans of non-consolidated sublitt oral of the São Vicente Estuarine Bay Complex, São Paulo State Brazil. Check List, 2(2), 84-88. https://doi.org/10.15560/2.3.84

Rhyne, A. L., \& Junda, L. (2006). A western Atlantic peppermint shrimp complex: redescription of Lysmata wurdemanni, description of four new species, and remarks on Lysmata rathbunae (Crustacea: Decapoda: Hippolytidae). Bulletin of Marine Science, 79(1),165-204. https://repository.si.edu/handle/10088/3627

Rieger, P. J. (1997). Os “ermitões” (Crustacea, Decapoda, Parapaguridae, Diogenidae e Paguridae) do litoral do Brasil. Nauplius, 5(2), 99-124.

Rodrigues, S. D. A., \& Shimizu, R. M. (1997). Autoecology of Callichirus major (Say, 1818). Oecologia Australis, 3(1), 155-170. https://revistas.ufrj.br/index.php/oa/article/view/5566

Romano, C., Fanelli, E., D'anna, G., Pipitone, C., Vizzini, S., Mazzola, A., \& Badalamenti, F. (2016). Spatial variability of soft-bottom macrobenthic communities in northern Sicily (Western Mediterranean): Contrasting trawled vs. untrawled areas. Marine environmental research, 122, 113-125. https://doi.org/10.1016/j.marenvres.2016.10.002

Sant'Anna, B. S., Watanabe, T. T., Turra, A., \& Zara, F. J. (2012). Relative abundance and population biology of the non-indigenous crab Charybdis hellerii (Crustacea: Brachyura: Portunidae) in a southwestern Atlantic estuary-bay complex. Aquatic Invasions, 347-356. https://repositorio.unesp.br/handle/11449/1243

Santos, J. (2020). Aspectos estruturais e funcionais da comunidade de crustáceos (Decapoda) da Baía De Santos-SP. [tese de doutorado, Universidade Federal de São Carlos, São Paulo, Brasil].

Santos, J. L., Severino-Rodrigues, E., \& Vaz-dos-Santos, A. M. (2008). Estrutura populacional do camarão-branco Litopenaeus schmitti (Burkenroad, 1936) nas regiões estuarinas e marinhas da Baixada Santista, São Paulo, Brasil. Boletim Instituto de Pesca, 34: (3), 375- 389. https://www.pesca.sp.gov.br/boletim/index.php/bip/article/view/807.

Scalco, A. C. S., Severino-Rodrigues, E., de Souza, M. R., Fagundes, L., Tutui, S. L. S., \& Tomás, A. R. G. (2018). Captura de siris pela comunidade da Vila dos pescadores (Cubatão) no estuário de Santos-São Vicente (SP). Boletim do Instituto de Pesca, 40(3), 389-395. https://www.pesca.sp.gov.br/40_3-389-395.pdf

Severino-Rodrigues, E., Guerra, D. S. F., \& Graça-Lopes, R. (2002). Carcinofauna acompanhante da pesca dirigida ao camarão-sete-barbas (Xiphopenaeus kroyeri) desembarcada na Praia do Perequê, Estado de São Paulo, Brasil. Boletim Instituto de Pesca, 28(1), 33-48. https://doi.org 10.20950/1678$2305.2016 \mathrm{v} 42 \mathrm{n} 3 \mathrm{p} 611$

Severino-Rodrigues, E., Musiello-Fernandes, J., Moura, Á. A. S., Branco, G. M. P., \& Canéo, V. O. C. (2012). Biologia reprodutiva de fêmeas de Callinectes danae (Decapoda, Portunidae) no complexo estuarino-lagunar de Iguape e Cananéia (SP). Boletim do Instituto de Pesca, 38(1), 31-41. https://www.pesca.sp.gov.br/boletim/index.php/bip/article/view/940>.

Severino-Rodrigues, E., Pita, J. B., \& Graça-Lopes, R. (2001). Pesca artesanal de siris (Crustacea, Decapoda, Portunidae) na região estuarina de Santos e São Vicente (SP), Brasil. Boletim do Instituto de Pesca, 27(1), 7-19. https://www.pesca.agricultura.sp.gov.br/arq_27_art_02.pdf

Silva, E. R. D., Sancinetti, G. S., Fransozo, A., Azevedo, A., \& Costa, R. C. D. (2014). Biodiversity, distribution and abundance of shrimps Penaeoidea and Caridea communities in a region the vicinity of upwelling in Southeastern of Brazil. Nauplius, 22(1), 1-11. https://doi.org/10.1590 S0104-64972014000100001

Simões, S. M. (2012). Estrutura da comunidade e biologia reprodutiva dos camarões marinhos (Penaeidea e Caridea), no Complexo Baía-Estuário de Santos e São Vicente/SP, Brasil. (tese de doutorado, Universidade Estadual Paulista Julio de Mesquita Filho). https://repositorio.unesp.br/handle/11449/106502

Simões, S. M., Heckler, G. S., \& Costa, R. C. (2017). Reproductive period and recruitment of Penaeoidea shrimp on the southeastern Brazilian coast: implications for the closed season. Crustaceana, 90(7-10), 1177-1192. https://doi.org/10.1163/15685403-00003664 
Research, Society and Development, v. 10, n. 1, e17210110682, 2021 (CC BY 4.0) | ISSN 2525-3409 | DOI: http://dx.doi.org/10.33448/rsd-v10i1.10682

Simões, S. M., Costa, R. C., Fransozo, A., \& Castilho, A. L. (2010). Diel variation in abundance and size of the seabob shrimp Xiphopenaeus kroyeri (Crustacea, Penaeoidea) in the Ubatuba region, Southeastern Brazil. Anais da Academia Brasileira de Ciências, 82(2), 369-378. https://doi.org/10.1590/S000137652010000200013

Siqueira, G. W., De Lima, W. N., Mendes, A. S., Aprile, F. M., Braga, E. D. S., \& Mahiques, M. M. (2012). Evolução do impacto ambiental causado por matéria orgânica, mercúrio e arsênio nos sedimentos de fundo do sistema estuarino de Santos. Geochimica Brasiliensis, 18(1), 54-63. http://doi.org/10.21715/gb.v18i1.211

Souza, M. R. de. (2017). Diversidade da ictio e carcinofauna do Sistema Estuarino de Santos-São Vicente (SP), Brasil: uma ferramenta para a avaliação da integridade ambiental. (tese de doutorado, Universidade Estadual Paulista Júlio de Mesquita Filho). https://repositorio.unesp.br/handle/11449/149923

Sousa, E. C. P. M., Zaroni, L. P., Gasparro, M. R., \& Pereira, C. D. S. (2014). Review of ecotoxicological studies of the marine and estuarine environments of the Baixada Santista (São Paulo, Brazil). Brazilian Journal of Oceanography, 62(2), 133-147. https://doi.org/10.1590/S1679-87592014063006202

Terossi, M., Almeida, A. O., Buranelli, R. C., Castilho, A. L., Costa, R. C., Zara, F. J. \& Mantelatto, F. L. (2018). Checklist of decapods (Crustacea) from the coast of the São Paulo state (Brazil) supported by integrative molecular and morphological data: I. Infraorder Caridea: families Hippolytidae, Lysmatidae, Ogyrididae, Processidae and Thoridae. Zootaxa, 4370(1), 76-94. https://doi.org/10.11646/zootaxa.4370.1.6

Tommasi, L. R. (1967). Observações preliminares sobre a fauna bêntica de sedimentos moles da Baía de Santos e regiões vizinhas. Boletim do Instituto Oceanográfico, 16(1), 43-65. http://doi.org/10.1590/S0373-55241967000100005

Vianna, M., \& Almeida, T. (2005). Bony fish bycatch in the southern Brazil pink shrimp (Farfantepenaeus brasiliensis and F. paulensis) fishery. Brazilian Archives of Biology and Technology, 48(4), 611-623. http://doi.org/10.1590/S1516-89132005000500014

WORMS - World Register of Marine Species. (2020). http://www.marinespecies.org/aphia.php?p=taxdetails\&id=585443 